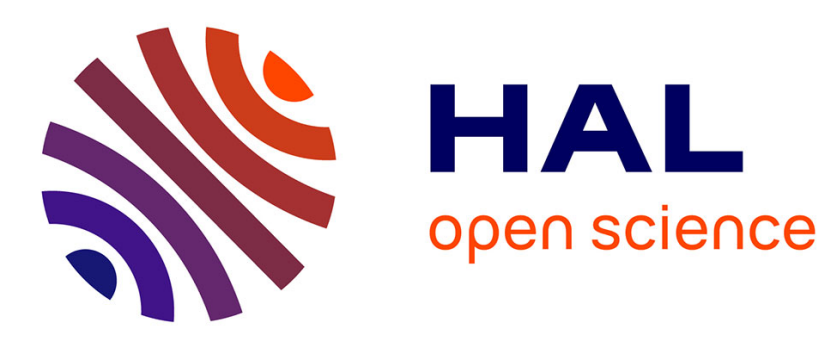

\title{
A review of the optical microstructure and rheology of carbon nanotube suspensions
}

\author{
A. W. K. Ma, K. M. Yearsley, Francisco Chinesta, M. R. Mackley
}

\section{To cite this version:}

A. W. K. Ma, K. M. Yearsley, Francisco Chinesta, M. R. Mackley. A review of the optical microstructure and rheology of carbon nanotube suspensions. Proceedings of the Institution of Mechanical Engineers, Part N: Journal of Nanoengineering and Nanosystems, 2010, 222 (3), pp.71-94. 10.1243/17403499JNN153 . hal-01004938

\section{HAL Id: hal-01004938 \\ https://hal.science/hal-01004938}

Submitted on 19 Feb 2017

HAL is a multi-disciplinary open access archive for the deposit and dissemination of scientific research documents, whether they are published or not. The documents may come from teaching and research institutions in France or abroad, or from public or private research centers.
L'archive ouverte pluridisciplinaire HAL, est destinée au dépôt et à la diffusion de documents scientifiques de niveau recherche, publiés ou non, émanant des établissements d'enseignement et de recherche français ou étrangers, des laboratoires publics ou privés. 


\title{
A review of the microstructure and rheology of carbon nanotube suspensions
}

\author{
A W K M a ${ }^{1}$, K M Yearsley ${ }^{1}$, F Chinesta ${ }^{2}$, and M R Mackley ${ }^{1}$ \\ ${ }^{1}$ Department of Chemical Engineering and Biotechnology, University of Cambridge, Cambridge, UK \\ ${ }^{2}$ EADS Corporate Foundation, CNRS - Centrale Nantes, Nantes, France
}

\begin{abstract}
The present review is concerned with the way that the incorporation of carbon nanotubes (CNTs) into a fluid matrix can modify the microstructure and rheology of the resulting suspensions. Some background to CNT manufacture and in particular methods of dispersing them into a suspension is presented for a range of different systems, where effective dispersion of CNTs remains a delicate and open issue. Steady shear, linear viscoelasticity, non-linear viscoelasticity and extensional responses are classified for a range of different $\mathrm{CNT} /$ matrix combinations together with their associated microstructure. The rheological modelling of certain $\mathrm{CNT} /$ matrix systems is reviewed, with particular attention given to the authors' work on modelling CNT suspension behaviour using Fokker-Planck advection-diffusion modelling.
\end{abstract}

Keywords: carbon nanotubes, suspensions, rheology, microstructure

\section{INTRODUCTION}

Carbon nanotubes (CNTs) are nano-scale fibres constituting an important part of the emerging nanotechnology field. The tubes may be singlewalled (SWNT) or multi-walled (MWNT). There are three main methods for producing CNTs: the arcdischarge method [1], the chemical vapour deposition method [2-4], and the laser ablation method [5]. Both the arc-discharge and laser ablation methods are energy-intensive involving evaporation of a graphite source, whereas chemical vapour deposition is based on the pyrolysis of gas-phase hydrocarbons in the presence of catalysts at elevated temperatures. Chemical vapour deposition is by far the most common method because of its advantages in offering controlled synthesis, continuous operation and scalability $[\mathbf{6}, 7]$. Thanks to advances in synthesis technology, CNTs are now available in gram, and increasingly kilogram, quantities [6, 7].

Although the description of CNT or CNT-like structures can be traced back to a much earlier date, the paper by Iijima published in 1991 [8] stimulated a lot of research interest in relation to the properties and applications of CNTs [9-12]. For many of the applications proposed, CNTs are dispersed in a medium, whether a solvent, a reagent, or a polymer. To this end, the nanotubes may be chemically treated to facilitate dispersion and prevent aggregation within the medium $[\mathbf{1 3}, \mathbf{1 4}]$. In some cases, further processing techniques such as fibre spinning, film casting, extrusion and inkjet printing may be applied to the composite material [15-19]. The properties of the resulting product may be very sensitive to the processing history $[\mathbf{2 0}, \mathbf{2 1}]$ and it is therefore important to understand the rheology as well as the microstructure of CNT suspensions.

Over the last decade, the optical microstructure and rheology of CNT suspensions have been studied by a number of researchers for different types of CNTs and suspending media with a wide range of base viscosities (see Tables 1 and 2). The present paper first gives an overview of the common methods used for dispersing CNTs into a fluid matrix. This is then followed by a review of key findings on the optical microstructure and rheology of CNT suspensions. In addition to experimental findings, the development of quantitative models that can describe the rheology of CNT suspensions is also presented. 
Table 1 Summary of optical microstructure studies on CNT suspensions

\begin{tabular}{llll}
\hline Nanotube & Suspending medium & External field & Reference \\
\hline SWNT & Oleum and 96\% sulfuric & - & {$[\mathbf{2 2}]$} \\
& acid & & \\
MWNT & Epoxy & Electric field & {$[\mathbf{2 3}]$} \\
& Synthetic lipid/water & Magnetic field & {$[\mathbf{2 4}]$} \\
& Epoxy & Shear flow & {$[\mathbf{2 5}]$} \\
& Phenoxy-ethyleneoxy/ & & {$[\mathbf{2 6}]$} \\
water & & {$[\mathbf{2 7}]$} \\
PB & & {$[\mathbf{2 8}],[\mathbf{2 9}]$} \\
PIB & - & {$[\mathbf{3 0}]$} \\
\hline
\end{tabular}

CNT, carbon nanotube; SWNT, single-walled carbon nanotube; MWNT, multi-walled carbon nanotube; PB, polybutadiene; PIB, polyisobutylene.

Table 2 Summary of rheological studies on CNT suspensions

\begin{tabular}{|c|c|c|c|}
\hline Nanotube & $\begin{array}{l}\text { Suspending } \\
\text { medium }\end{array}$ & $\begin{array}{l}\text { Steady shear } \\
\text { (model fitting) }\end{array}$ & LVE Reference \\
\hline \multirow[t]{4}{*}{ SWNT } & PMMA & & {$[31]$} \\
\hline & PS & & {$[32]$} \\
\hline & $\begin{array}{l}\text { Sodium dodecyl } \\
\text { benzenesulfonate/ } \\
\text { water }\end{array}$ & & {$[33]$} \\
\hline & $\begin{array}{l}\text { Oleum and } 96 \% \\
\text { sulfuric acid }\end{array}$ & & $\checkmark \quad[22]$ \\
\hline \multirow[t]{11}{*}{ MWNT } & Epoxy & & {$[34]$} \\
\hline & Epoxy & $\checkmark$ (Cross) & {$[25]$} \\
\hline & $\begin{array}{l}\text { Polyacryl/ } \\
\text { polyaspartic acid }\end{array}$ & $\checkmark$ & {$[\mathbf{3 5}]$} \\
\hline & PA6 & & {$[36]$} \\
\hline & $\mathrm{PC}$ & & {$[37]$} \\
\hline & PDMA & & {$[\mathbf{3 8}]$} \\
\hline & $\mathrm{PE}$ & & {$[39]$} \\
\hline & PIB & $\checkmark$ & {$[40]$} \\
\hline & $\mathrm{PP}$ & $\checkmark$ (Carreau) & {$[41]$} \\
\hline & PS & $\checkmark$ & {$[16]$} \\
\hline & Water & $\begin{array}{l}\text { (Bingham and } \\
\text { Herschel-Buckley) }\end{array}$ & $\checkmark \quad[42]$ \\
\hline
\end{tabular}

CNT, carbon nanotube; SWNT, single-walled carbon nanotube; MWNT, multi-walled carbon nanotube; LVE, linear viscoelasticity; PMMA, poly(methyl methacrylate); PS, polystyrene; PA6, polyamide 6; PC, polycarbonate; PDMA, polydimethylsiloxane; PE, polyethylene; PIB, polyisobutylene; PP, polypropylene.

\section{HISTORY}

Owing to difficulties in manufacturing large quantities of CNTs in the early days of their development, rheological studies were performed using carbon nanofibres as a precursor to CNTs $[\mathbf{4 3}, 44]$. Experimental studies on the rheology of CNT suspensions were reported as early as 2002. Many of the early rheological experiments on CNT/thermoplastic systems have been reported by Dr Petra Pötschke from the Leibniz Institute of Polymer Research Dresden, including the first linear viscoelastic experiments [37] and the first investigations into their extensional rheology [45]. The first steady shear experiment on a $\mathrm{CNT} /$ polymer nanocomposite was reported by Safadi et al. [16] where MWNTs were dispersed in toluene and the resulting suspensions were mixed with toluene solutions of $30 \mathrm{wt} \%$ polystyrene. The viscosities of the mixtures with various MWNT contents were measured at room temperature over a range of shear rates. Around the same time, Kinloch et al. [42] reported the experimental rheology results for concentrated aqueous CNT dispersions, covering the steady shear, linear viscoelasticity (LVE), and recovery after pre-shear behaviour of these dispersions.

\section{DISPERSION OF CNTs}

\subsection{Background}

The formulation of homogeneous CNT suspensions has proved to be a very difficult task which has not yet been completely resolved. CNTs tend to agglomerate and bundle due to van der Waals forces, and mechanically mixing CNT into a liquid does not necessarily produce a homogeneous suspension [46]. The issue has been studied in some detail by, for example, Huang et al. [38], Fan and Advani [47], and Hong and Kim [48]. The formation of a homogeneous suspension of CNT involves separating individual CNTs from the bundles as well as stabilizing the 'debundled' CNTs to prevent re-aggregation. Some of the common dispersion methods for CNTs are reviewed and discussed in the following sections. However, the results of mixing generally depend on a number of variables such as the initial entanglement state of the CNT and the mixing unit design, and it is therefore difficult to identify a unique set of optimal mixing conditions for all CNT systems [49].

\subsection{Common methods of dispersion}

High-shear mechanical mixing and sonication are two of the most common methods for dispersing CNTs into a suspending medium having a relatively low viscosity [46]. In high-shear mixing, high shear is created within a small gap between an impeller and a stator. High-shear mixers are commercially available and have been used for preparing emulsions. Dispersion by sonication, on the other hand, relies on cavitation (the implosion of small bubbles generated in the suspending medium) occurring within the sample [46]. As the bubbles implode, large amounts of energy are released locally to break up the aggregates and to disrupt highly entangled CNTs. Sonication probes or horns that generate high power intensity, sometimes known as 'cell disrupters' [50], have been widely used [46]. For blending CNTs into matrices with high viscosity such as thermoplastics, 
twin-screw mixers are commonly employed [37, 41, 51].

Although there has been some success in using high-shear mixing and sonication to achieve a fairly uniform dispersion, these high-intensity mixing methods were found to shorten the length of CNTs and therefore adversely affect product performance for applications that benefit from the high aspect ratio of CNTs. The effect of prolonged sonication on the length of CNTs has been studied experimentally $[\mathbf{5 2}, \mathbf{5 3}]$. The key observation is that, for fixed sonication conditions, there exists a limiting length beyond which the CNTs will not be further shortened. Huang and Terentjev [46] constructed a simple model to calculate the 'limiting length'; theoretically, there should be an optimal sonication condition (in terms of sonication power, frequency, and time) that would allow the dispersion of CNTs without shortening their length significantly.

\subsection{Stabilizing CNT dispersions}

Once the CNTs are dispersed, it is of equal importance to stabilize them to prevent re-aggregation. To this end, chemical treatment, also known as functionalization, can be used [13, 14]. Functional groups are attached, covalently or non-covalently, onto the walls or caps of the CNTs, with the aim of disrupting or weakening the natural attraction between them (see reviews by Banerjee et al. [54], Balasubramanian and Burghard [55], and Prato et al. [14]). Among all covalent functionalization techniques, refluxing CNTs in strong acid mixtures is one of the most common methods as it can simultaneously remove impurities and introduce carboxylic acid groups onto the walls of CNTs, thereby rendering the CNTs soluble in water [42]. In the case of noncovalent functionalization, surfactants such as sodium dodecyl sulfate, Triton X-100, and dye molecules such as porphyrin and phthalocyanines are commonly used [56-58].

\section{MICROSTRUCTURE OF CNT SUSPENSIONS}

The quality of dispersion can be evaluated by characterizing the microstructure of CNT suspensions after dispersion. In the literature, the microstructure of CNT suspensions has been studied by a number of authors for different types of nanotubes and suspending media, sometimes in the presence of external fields. A summary of optical microstructure studies on CNT suspensions is given in Table 1; some of the key findings are highlighted and discussed in the present paper.

\subsection{Quiescent optical microstructure}

\subsubsection{Microstructure after mixing}

Figure 1 shows optical microstructures observed for four different concentrations of untreated CNTs suspended within an optically transparent epoxy [59]. The suspensions were prepared using a high-shear mechanical mixing method as reported in reference [60]. For low concentration samples, a clear network of CNT can be resolved (Figs 1(a) to (c)) whereas the 0.5 wt\% concentration sample (Fig. 1(d)) showed domains of highly aggregated CNTs.

Confocal microscopy has also been used to characterize the microstructure of CNT suspensions after mixing by sonication (Fig. 2). MWNTs, $0.03 \mathrm{wt} \%$, were dispersed in epoxy using a Sonicbio NR-3000 ultrasonic bath. The sonicator was run with a cycle consisting of $30 \mathrm{~s}$ of ultrasound followed by $30 \mathrm{~s}$ of rest. Samples were taken for imaging after $5 \mathrm{~min}$ (Fig. 2(a)) and 60 min (Fig. 2(b)) of treatment. They were imaged using a Leica TCS SP5 microscope. Light of $633 \mathrm{~nm}$ wavelength was shone at the sample, and transmitted light and light reflected at the same wavelength were detected. Quantitative analysis has not yet been performed on the average size of structure in each sample, but it is possible to qualitatively conclude that the structures after $60 \mathrm{~min}$ of sonication (Fig. 2(b)) are smaller than those after $5 \mathrm{~min}$ (Fig. 2(a)).

\subsubsection{Nematic phase formation at high concentration}

A number of research groups have studied the effect of CNT concentration on the microstructure of CNT suspensions and have observed the formation of a nematic phase at high concentration levels, where the CNTs have long-range orientational order and only short-range positional order [61]. For instance, Professor Alan Windle and his group at the University of Cambridge dispersed some acid-treated MWNTs in water and observed a phase transition from an isotropic phase to a nematic phase at a concentration of $4.3 \mathrm{vol \%}$ [30]. At Rice University, Professor Matteo Pasquali and co-workers [22] discovered that CNTs can be protonated and remain dispersed in superacids such as fuming sulfuric acid and chlorosulfonic acid. Although similar phase transition behaviour has been reported for the tobacco mosaic virus [62], these experimental observations could have farreaching implications in terms of processing CNT suspensions into products with a high degree of CNT alignment. It has been demonstrated by the Rice group that CNT fibres, as shown in Figs 3(b) and (c), can be spun from a liquid crystalline solution of CNTs in superacids (Fig. 3(a)). 

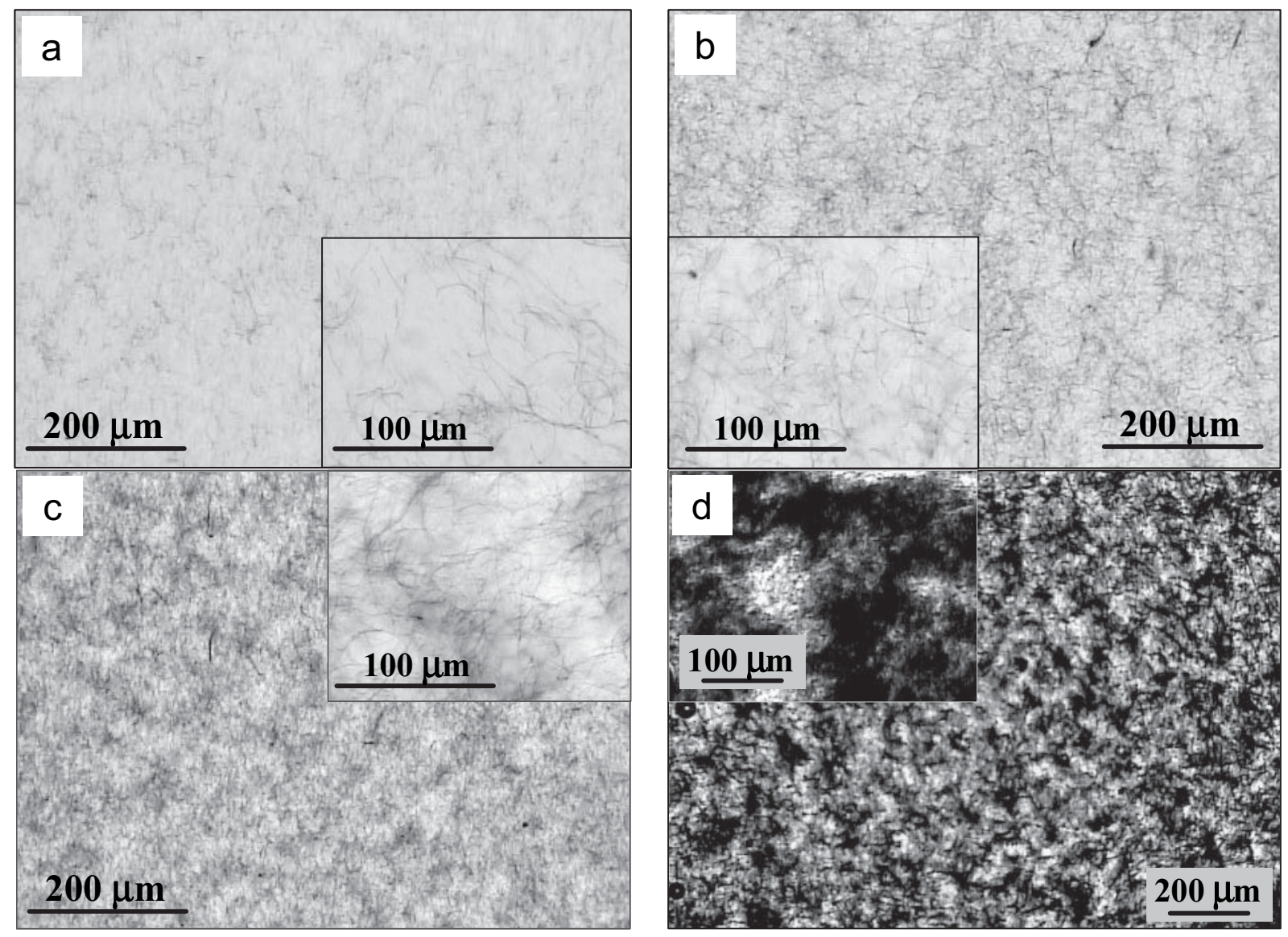

Fig. 1 Optical micrographs of different concentrations of untreated MWNT suspended in epoxy: (a) $0.025 \mathrm{wt} \%$, (b) $0.05 \mathrm{wt} \%$, (c) $0.1 \mathrm{wt} \%$, and (d) $0.5 \mathrm{wt} \%$. Optical depth $=130 \mu \mathrm{m}$; temperature $=$ $25{ }^{\circ} \mathrm{C}$ [59]
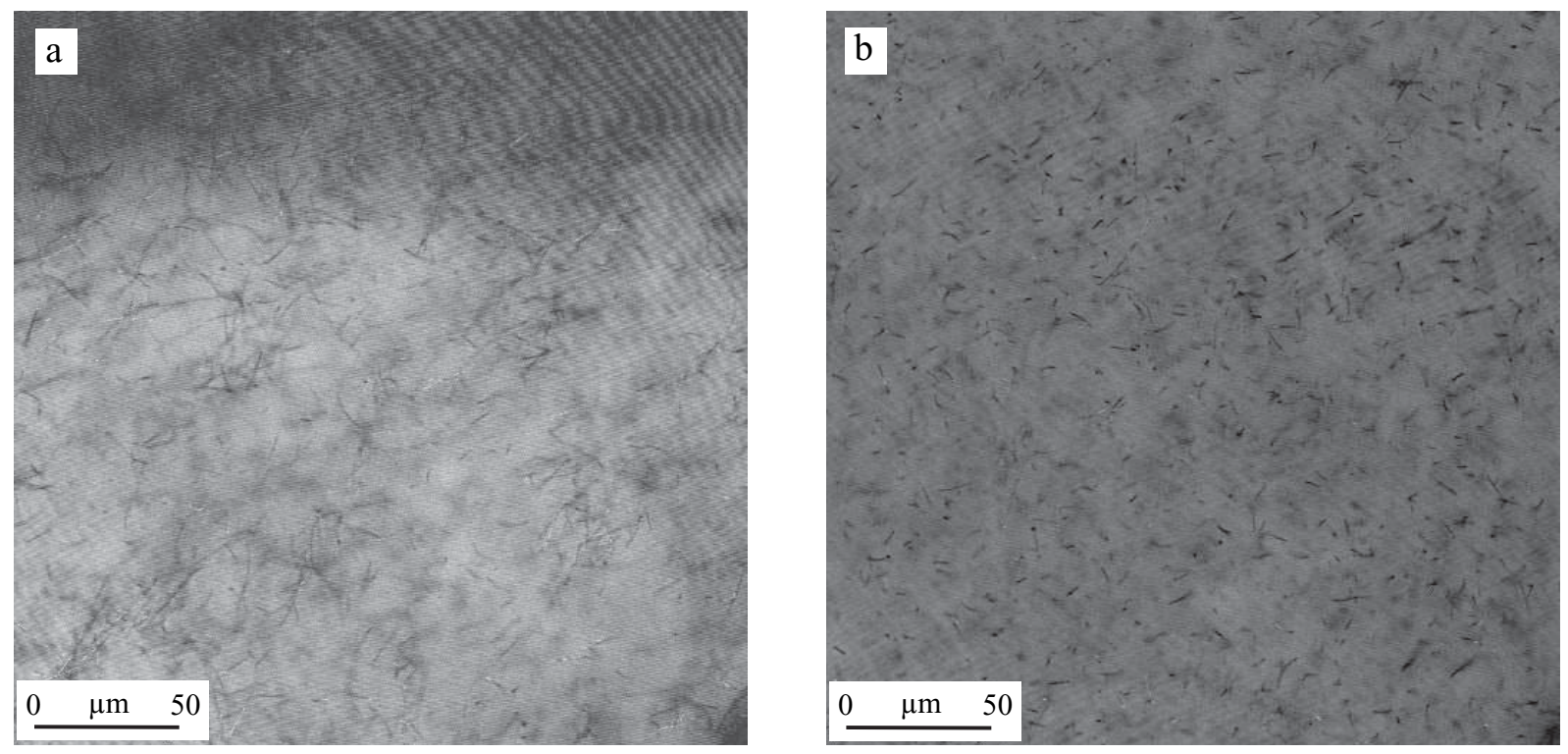

Fig. 2 Confocal images of $0.03 \mathrm{wt} \%$ MWNT suspended in epoxy after (a) $5 \mathrm{~min}$ and (b) 60 min of mixing by sonication 

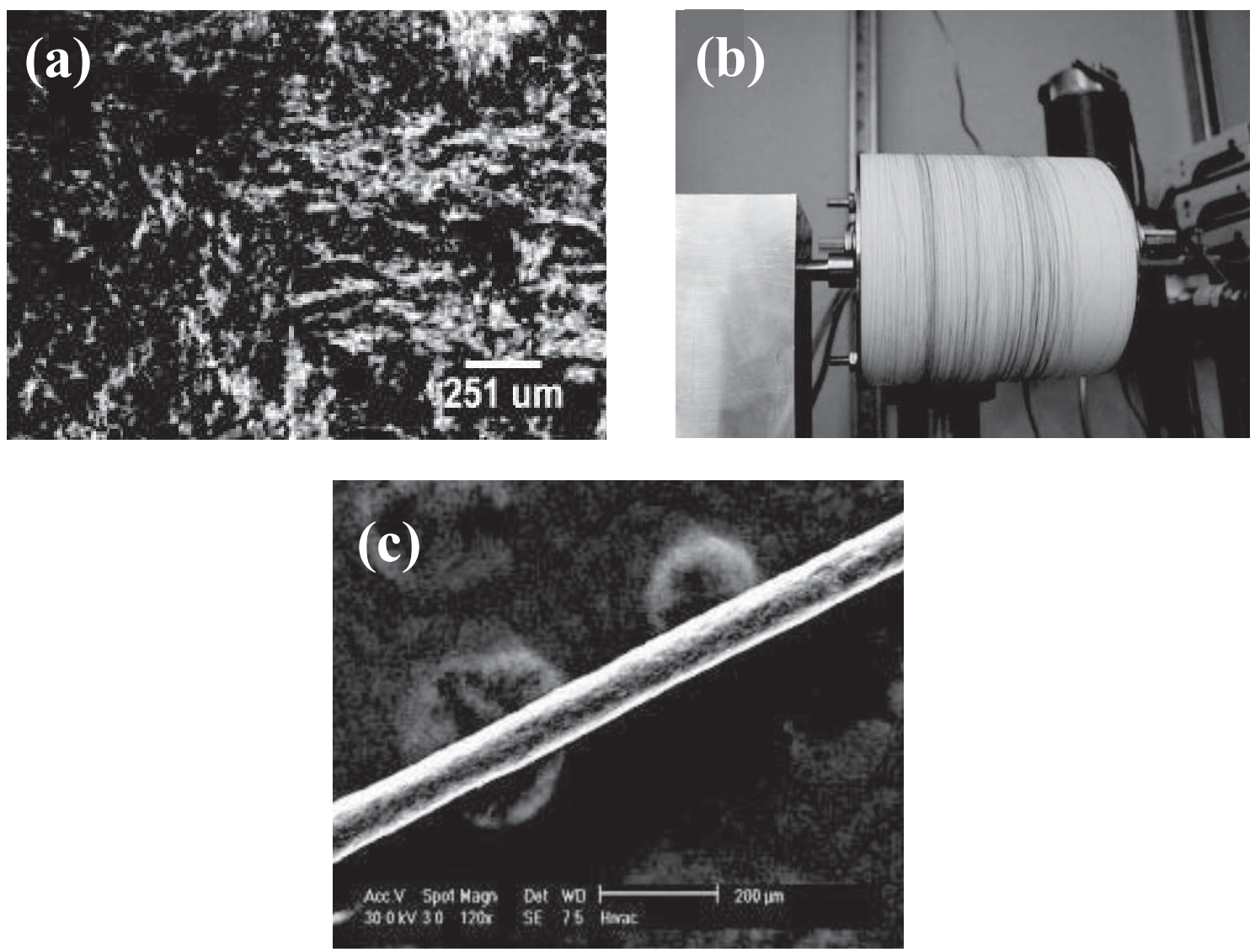

Fig. 3 (a) A liquid crystalline solution containing 4 wt\% of CNTs dispersed in $102 \mathrm{wt} \%$ sulfuric acid. The image was captured using cross-polarized light on an optical microscope. (b) A $30 \mathrm{~m}$ spool of water-coagulated fibre. (c) Scanning electron micrograph of a neat CNT fibre spun from a CNT/ superacid mixture. Reproduced from references [17] and [22]. Reprinted with permission from AAAS and ACS

\subsection{Microstructure formation in the presence of an external field}

\subsubsection{Shear flow}

The evolution of CNT microstructure in the presence of shear flow has been reported by a number of authors $[\mathbf{2 5 - 2 8 , 6 3}]$. The general observation is that low shear induces aggregation of CNTs whereas high shear breaks up CNT aggregates and subsequently aligns CNTs in the shear direction. Hobbie and Fry [29] characterized microstructures formed within $\mathrm{CNT}$ /polyisobutylene (PIB) suspensions for a range of CNT concentrations, gap sizes, and shear rates, and further classified the CNT microstructure as 'cavitated networks', vorticity bands, isolated aggregates, and 'flowing nematics'. It is worth noting that the shear-induced aggregation phenomenon is not unique to CNT suspensions and has also been reported for cellulose fibre suspensions, being referred to as 'flocculation'. Mason $[\mathbf{6 4 , 6 5}]$ suggested that flocculation of non-Brownian fibres is a result of mechanical entanglement as fibres rotate and collide in shear flow. In terms of modelling the shear- induced aggregation of fibres, Switzer and Klingenberg [66] performed particle-level simulations and proposed that fibres can flocculate via frictional contacts between fibres even in the absence of attractive forces.

For systems where the CNTs were suspended in a low-viscosity matrix, an unusual form of CNT aggregate structures, named 'helical bands', was observed for certain flow conditions. Figure 4 shows the formation of such structures reported by $\mathrm{Ma}$ and coworkers $[\mathbf{6 3}]$ as simple shear was applied to a CNT suspension confined within a small gap. The images were captured using a Cambridge Shear System, where rotary shear was applied to a CNT suspension confined between two quartz plates and optical observations were made at a distance from the centre of rotation, as shown in Fig. 5. It can be seen that the helical bands are highly anisotropic structures which align in the flow vorticity direction.

Figures 4(a) to (c) are optical images showing the evolution of microstructure as a function of time $t$. Initially, at $t=0$, diffuse CNT aggregates were present. As simple shear was applied, there was a 

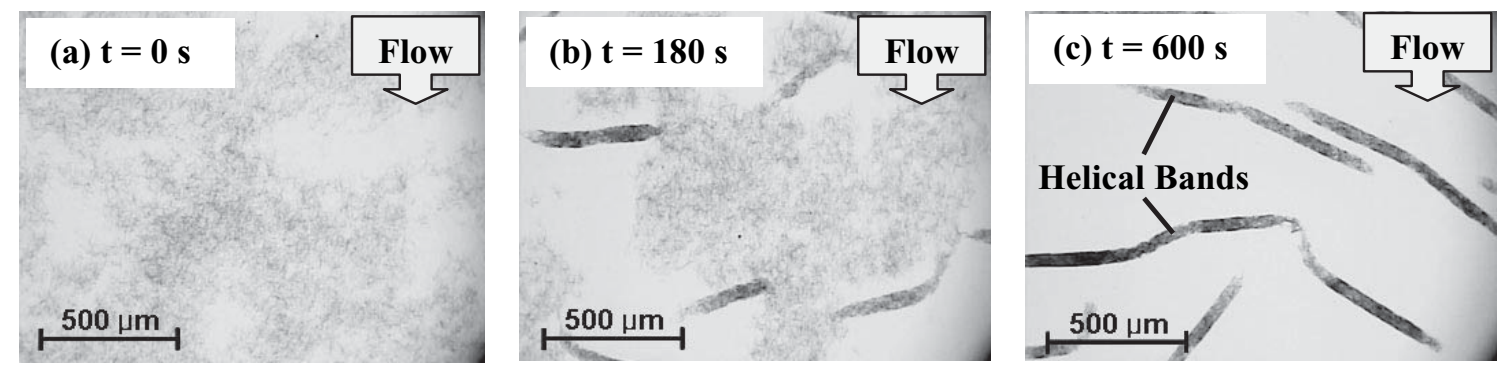

Fig. 4 Time evolution of optical microstructure as low shear $\left(0.5 \mathrm{~s}^{-1}\right)$ was applied to $0.03 \mathrm{wt} \%$ untreated MWNTs suspended in a low-viscosity epoxy (UV 60-7155) confined within a gap of $130 \mu \mathrm{m}$. Helical band structures were identified as shown in (c). Temperature $=25^{\circ} \mathrm{C}[\mathbf{5 9}, \mathbf{6 3}]$

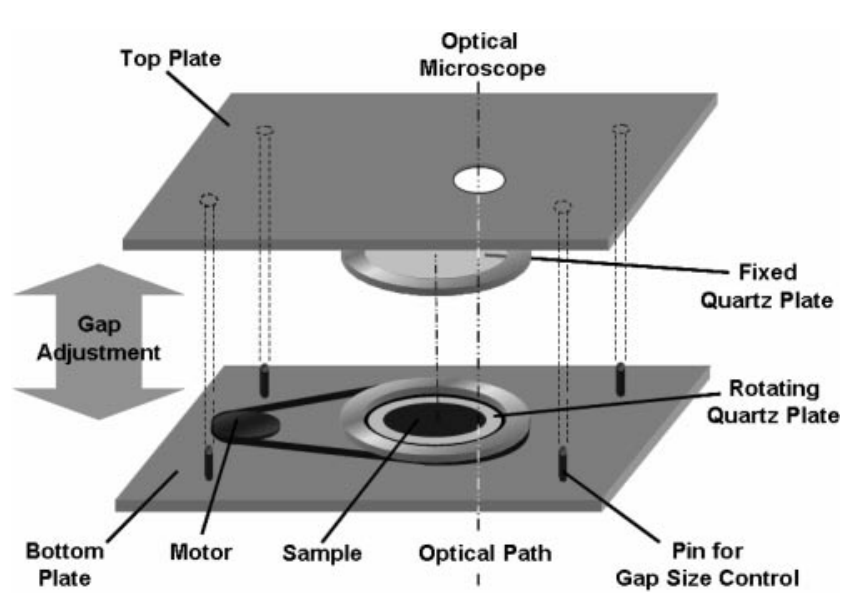

Fig. 5 The Cambridge Shear System (CSS450, Linkam Scientific Instruments) consists of a shear cell and an optical microscope. The sample was confined between two quartz plates and the bottom plate was connected to a motor. The gap size was varied between 55 and $230 \mu \mathrm{m}$. Steady rotational shear was applied to the sample via the bottom plate and microstructure formation in the sample was viewed using the optical microscope [63]

velocity gradient and these diffuse aggregates translated at different speeds, interlocked, and formed denser aggregates. These denser aggregates rotated in the simple shear flow and as they rotated, they captured the aggregates nearby and grew in both mass and length. The mechanism by which these highly anisotropic aggregate structures grow has similarity to the hand-spinning of cotton into fibres and the resulting structures were found to be stable at low shear conditions only. The formation of helical band structures was believed to be a result of mechanical aggregation and wall confinement where the small gap size prevented the CNT aggregates from growing as a spherical entity. Furthermore, direct observations also suggested that the rotation component inherent to a simple shear flow played an important role in the formation of these 'helical band' structures.

The way in which weak simple shear flow induces aggregation is best viewed in video mode, accessible as electronic supplementary material of Ma et al. [63]. The experimental observation of helical band and other types of aggregate structures has particular relevance to the processing of CNTs. Schmidt et al. [67] spin-coated suspensions of untreated CNTs suspended in poly(methyl methacrylate) (PMMA) and presented optical evidence of CNT aggregation. They suggested that there was a velocity gradient within the CNT/polymer film during the spin-coating process resulting in the interlocking and aggregation of the CNTs via a 'carpet sweeping' mechanism, consistent with the in situ observation reported by Ma et al. [63].

\subsubsection{Electric field}

In addition to shear flow, the presence of an electric field was able to align CNTs in various liquid media such as water, ethanol and isopropyl alcohol [68-73]. Martin et al. [23] applied both alternating-current (AC) and direct-current (DC) electric fields during the curing of CNT/epoxy composites and studied the formation of the CNT network structure using in situ optical microscopy. In their study, an AC electric field was found to be more effective in aligning CNTs and forming a more uniform network structure, while in a DC electric field, the CNT network formed was denser near the anode.

\subsubsection{Magnetic field}

Several researchers have demonstrated that a magnetic field is capable of aligning CNTs [74, 75], although the contribution from ferro/paramagnetic catalyst impurities still remains debatable [76, 77]. To enhance the magnetic susceptibility of CNTs, some researchers strategically incorporated paramagnetic or ferromagnetic materials using various chemical and physical methods as reviewed by Samouhos and McKinley [78]. Toledo and co-workers [24], for example, studied pattern formation during the drying of an aqueous CNT/lipid suspension and showed that aggregate structures were altered in the presence of a magnetic field. 


\subsection{Characterization of CNT orientation}

In addition to aggregate structures, characterizing CNT orientation is desirable as the orientation distribution of CNTs is closely related to properties such as the electrical conductivity of the system [21]. CNTs were found to absorb light strongly [79] and LinGibson et al. [28] suggested that individual CNTs, having a diameter as small as $50 \mathrm{~nm}$, are still discernible in bright-field optical microscopy provided that the length of the CNT is larger than the diffraction limit of the optics. In some cases, characterization of CNT orientation has proved to be more challenging [80] and requires the use of techniques such as small-angle neutron scattering. Other characterization techniques reported in the literature include polarized Raman microscopy [81-83], X-ray diffraction $[\mathbf{8 4}, \mathbf{8 5}]$, near-infrared fluorescence microscopy [86], and tagging CNTs with fluorescent markers [87-90]. Besides in situ characterization, the orientation distribution of CNTs can also be characterized indirectly by performing electron microscopy on extruded or cured samples (see, for example, references [24], [47], [91], and [92]).

\section{EXPERIMENTAL RHEOLOGY OF CNT SUSPENSIONS}

\subsection{Steady shear rheology}

The steady shear rheology of CNT suspensions has been studied and reported by a number of researchers as summarized in Table 2. In general, the addition of CNTs into a suspending medium was found to increase the base viscosity of the whole system but this viscosity enhancement effect decreased as a function of increasing shear rates, as shown in Fig. 6. The decreasing trend of viscosity as a function of increasing shear rates is also known as 'shear thinning' and such behaviour has been reported for other types of suspension systems [93]. Although such shear-thinning behaviour is not unique to CNT suspensions, it is worth noting that some CNT suspensions tended to show a much stronger shearthinning effect compared with traditional glass or fibre suspensions at similar concentration levels [37]. This observation could have an important implication on the processability of CNT suspensions as the addition of a small amount of CNTs could result in an increase of several orders of magnitude in shear viscosity, possibly making processing more difficult [25]. Some authors attributed the more pronounced effect to the comparatively high aspect ratio of the CNTs [47], whereas Rahatekar et al. [25] correlated their rheological findings with optical observation and conjectured that the significant viscosity enhancement effect observed at low shear rates was due to the interconnection of CNT aggregates within their untreated CNT suspensions.

To describe the experimental data of shear thinning, a number of empirical models, such as the Cross model, the Carreau model, and the KriegerDougherty model, have been used [25, 35, 41]. More recently, Ma and co-workers $[\mathbf{6 0}, \mathbf{9 4}]$ successfully modelled both aggregating and non-aggregating CNT suspensions using a stress-microstructure approach

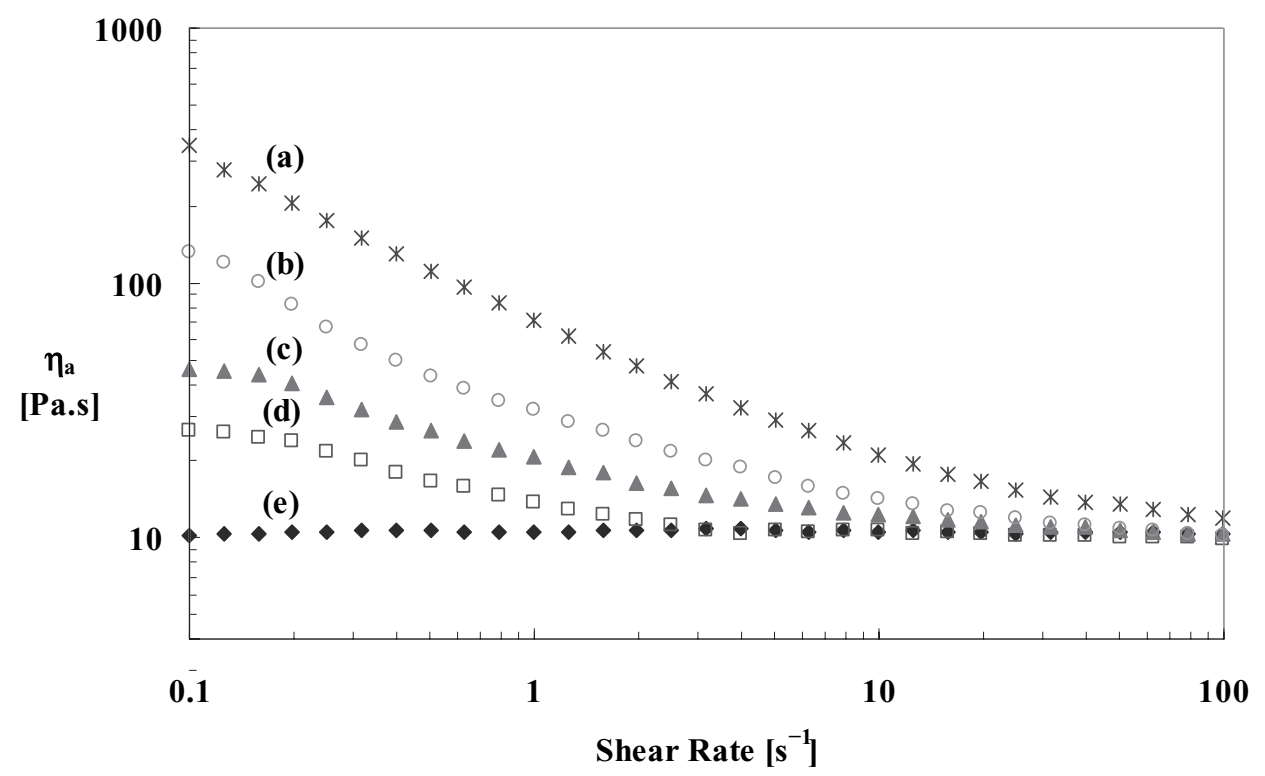

Fig. 6 Apparent shear viscosity $\eta_{\mathrm{a}}$ as a function of shear rate for suspensions containing (a) $0.25 \mathrm{wt} \%$, (b) $0.1 \mathrm{wt} \%$, (c) $0.05 \mathrm{wt} \%$, and (d) $0.025 \mathrm{wt} \%$ CNT, and (e) epoxy only. The $\eta_{\mathrm{a}}$ data presented are experimental data and the values were obtained after a shearing time of $100 \mathrm{~s}$ [60] 
that relates the CNT orientation and aggregation state with the overall viscosity of the system. Further discussions on detailed modelling of CNT suspensions can be found in section 7 .

\subsection{Linear viscoelasticity}

In addition to steady shear rheology, LVE studies are useful as they provide insight into how a material behaves at different processing time scales. In linear viscoelastic measurements, small-amplitude oscillations are applied to the sample, where the strain is written as

$$
\gamma=\gamma_{0} \sin \omega t
$$

where $\gamma_{0}$ is the maximum strain amplitude and $\omega$ is the angular frequency.

Stress $\tau$ is then measured and is represented as

$$
\tau(t)=\gamma_{0} G^{\prime} \sin \omega t+\gamma_{0} G^{\prime \prime} \cos \omega t
$$

where $G^{\prime}$ is the storage modulus and $G^{\prime \prime}$ is the loss modulus. The first term represents the elastic stress component that is in phase with the strain, whereas the second term corresponds to the viscous component that is in phase with the strain rate. Complex viscosity is defined as

$$
\eta^{*}=\sqrt{\left({G^{\prime}}^{2}+G^{\prime 2}\right)} / \omega
$$

Compared to steady shear experiments, there have been more studies focusing on the LVE of CNT suspensions, as summarized in Table 2. The general observation is that addition of CNTs increased both the values of storage modulus $G^{\prime}$ and loss modulus $G^{\prime \prime}$ but the effect on $G^{\prime}$ was generally larger $[\mathbf{3 7}, \mathbf{3 9}]$. A $G^{\prime}$ plateau at low frequencies was usually observed at high CNT loadings, indicating the presence of a physical network of CNTs (aggregates) [36, 41, 95].

In an attempt to understand the LVE of a surfactant-stabilized CNT suspension, Hough et al. [33] performed scaling analysis and suggested that the elasticity observed experimentally was probably due to interaction between the surfactant-stabilized CNTs and not because of bending or stretching of CNTs. More recently, Hobbie and Fry [40] reported similar scaling analysis for MWNTs suspended within a Newtonian PIB fluid. The MWNTs were stabilized with a polymer dispersant but could aggregate at low shear conditions. From their scaling analysis, the authors suggested that the MWNTs had formed an elastic network which contributed to both steady shear and LVE response. Despite earlier efforts in scaling analysis, detailed LVE modelling was found to be more challenging and further comments are given in section 7 .

\subsection{Non-linear viscoelasticity}

\subsubsection{Stress relaxation after step strain}

A series of step strain experiments was carried out by Ma [59] to characterize the relaxation behaviour of CNT suspensions. In a step strain experiment, a finite step strain $\gamma_{\mathrm{s}}$ is applied to the CNT suspension and the stress is then recorded as a function of time. The relaxation modulus is defined as

$$
G(t)=\tau(t) / \gamma_{\mathrm{s}}
$$

Depending on the stress relaxation pattern, step strain experiments provide essential information concerning the percolation threshold for a CNT suspension. The theoretical background of percolation is first reviewed before the experimental results for CNT suspensions are presented.

\subsubsection{Percolation threshold and stress relaxation behaviour}

Classic work on percolation can be traced back to the early 1940s in understanding the gelation process for polymeric systems [96-98] and subsequent percolation theory can be found in the work of Stauffer [99] and de Gennes [100]. Physical percolation, or physical gelation, can be defined as the growth of physically connected aggregates and a physical 'percolation threshold' is reached when the correlation length of molecular (or supramolecular) motion diverges to infinity [101]. Winter and co-workers [101-104] showed that at the percolation threshold, the relaxation modulus $G(t)$ can be written as

$$
G(t)=s t^{-n}
$$

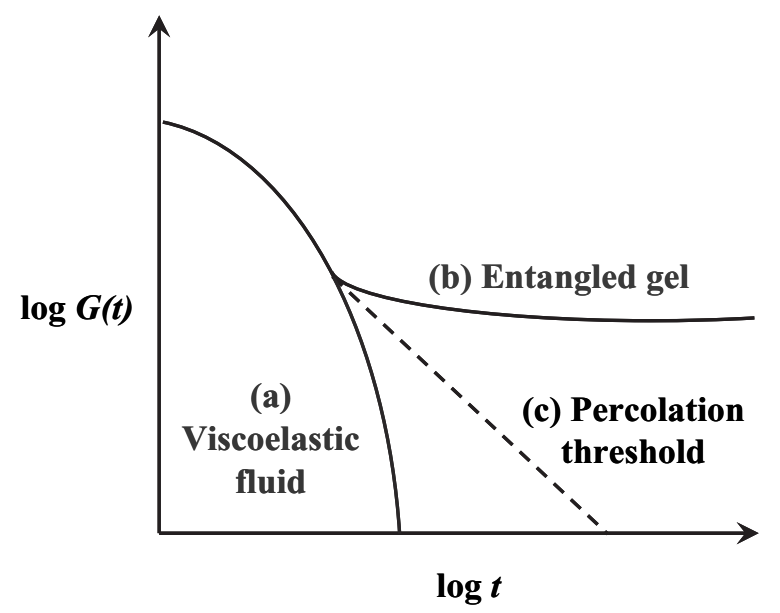

Fig. 7 Schematic diagram showing three typical types of relaxation response in step strain experiments: (a) viscoelastic fluid response, (b) entangled gel response, (c) percolation threshold. The plot is a $\log -\log$ plot of the relaxation modulus $G(t)$ versus time $t[101,106]$ 
where $s$ is a parameter of the gel stiffness and $n$ is the relaxation exponent. Graphically, the equation represents a straight line on a log-log plot as shown in Fig. 7 [101, 105].

The stress relaxation for untreated CNT suspensions was characterized by $\mathrm{Ma}[\mathbf{9 5}]$ and the results are shown in Fig. 8. Epoxy was the suspending medium and Fig. 8(a) shows the stress relaxation results for epoxy subjected to different magnitudes of step strain. Both steady shear and LVE measurements indicated that the epoxy matrix behaved essentially as a Newtonian fluid with negligible elasticity. Theoretically, one would expect an instantaneous dissipation of stress for a Newtonian fluid, but in the actual experimental setting it generally took about $0.1 \mathrm{~s}$ (according to logged strain data) for the motor to respond and finally attain the strain magnitude specified. Motor response time has been taken into account in data analysis and marked by a dotted line in all of the experimental results presented. As shown in Fig. 8(a), the stress within the test epoxy sample dissipated in about $0.1 \mathrm{~s}$, matching the motor response time, and this observation further confirmed that epoxy behaved as a viscous fluid.

Figures 8(b) to (f) show the stress relaxation behaviour for five different concentrations $(\mathrm{w} / \mathrm{w})$ of untreated CNT suspensions. In terms of precautions for sample testing, a fresh sample was used in each
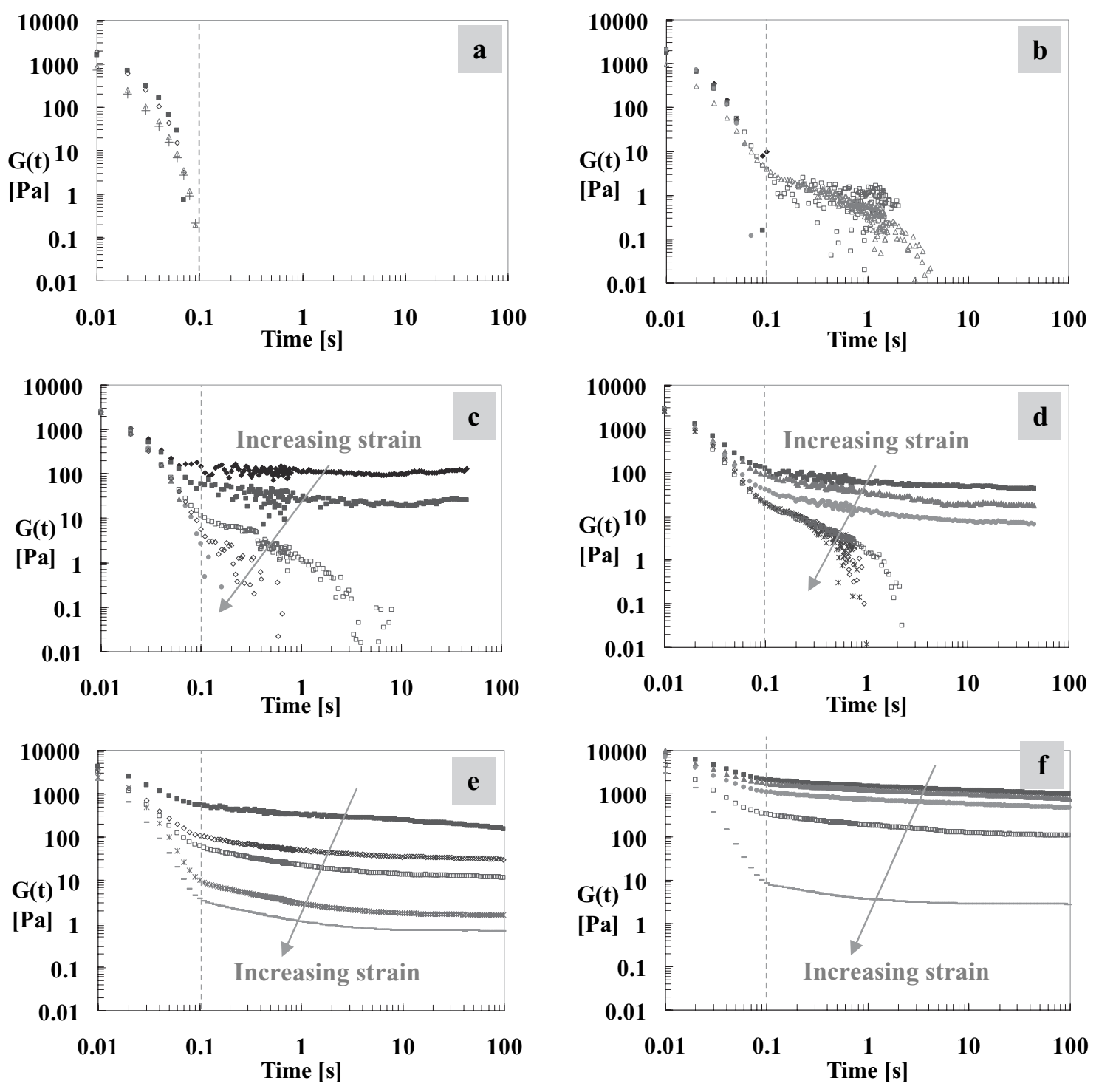

Fig. 8 Stress relaxation of (a) epoxy, (b) $0.025 w t \%$, (c) $0.05 w t \%$, (d) $0.1 w t \%$, (e) $0.25 w t \%$, and (f) $0.5 w t \%$ untreated MWNTs in epoxy after application of different magnitudes of step strain: 0.5 per cent $(\diamond), 1$ per cent $(\bullet), 2$ per cent $(\triangleleft), 5$ per cent $(\bullet), 10$ per cent $(\diamond), 20$ per cent $(\square), 100$ per cent $(\triangleleft), 200$ per cent (+), 500 per cent (ж), and 1000 per cent $(-)$. Arrows indicate increasing strain direction [59] 
experimental run to avoid complication from shear history and the sample was confined within the parallel-plate geometry by bringing down the upper plate slowly and limiting the maximum allowable force during sample loading. Adding CNTs to epoxy was found to prolong the stress relaxation time of the system. At a concentration of $0.025 \mathrm{wt} \%$, the suspension behaved as a viscoelastic fluid with a relaxation time in the order of seconds (Fig. 8(b)). At higher concentrations of 0.05 and $0.1 \mathrm{wt} \%$, the suspensions behaved either as a viscoelastic fluid or an entangled gel depending on the strain magnitude applied (Figs 8(c) and (d)). At small strains, these suspensions responded as an entangled gel and at large strains, decay characteristic of a viscoelastic fluid was observed. Intuitively, the strain dependence can be explained by the yielding of a physical network [107, 108]. If a large strain is applied, the network breaks down and finally dissipates like a fluid. Since the stress dissipation mode at small and large strains is different, the relaxation modulus cannot be factorized into the product of two independent functions of strain and time and the damping factor is thus not readily available [109-111]. For the suspensions containing 0.25 and $0.5 \mathrm{wt} \%$ of untreated MWNTs, an entangled gel response was observed for a strain of up to 1000 per cent. The suspensions also demonstrated a strain-softening behaviour where the stress stored within sample decreased as the magnitude of strain increased.

Different concentrations of untreated MWNT suspension were tested using different magnitudes of step strain and experimental results similar to those shown in Fig. 8 were summarized to construct a phase diagram (Fig. 9). As shown in the diagram, the epoxy matrix showed a viscous fluid dissipation for all strain conditions tested, whereas the untreated MWNT suspensions behaved as a viscoelastic fluid or an entangled gel, depending on the strain magnitude and concentration level. The transition from viscoelastic fluid behaviour to behaviour indicative of an entangled gel signified the percolation threshold as shown in Fig. 9. It is clear from the phase diagram that the percolation threshold depended on the CNT concentration as well as the magnitude of strain applied during testing. These experimental findings confirmed the conceptual model proposed by Mours and Winter [105] for physical gels and, more importantly, showed that stress relaxation after step strain could be a useful tool for identifying the physical percolation threshold of CNT suspensions.

\subsection{Extensional rheology}

Being able to understand the extensional rheology is important in terms of understanding the processing behaviour of CNT suspensions in fibre spinning [22], inkjet printing [19, 112], and curtain coating [113]. In the case of carbon nanofibres (CNFs), which are analogous to CNTs, Xu et al. [114] measured the extensional viscosity of some untreated CNFs suspended in a glycerol/water solution. They found that the extensional viscosity decreased as the extensional rate increased and this was probably due to the breakup of CNF network structures. Handge and Pötschke [45] performed elongation tests on polycarbonate melts containing CNTs and reported that the addition of untreated CNTs had little influence on the extensional viscosity. According to transmission electron microscopy (TEM) results, CNT clusters were present and CNT alignment in the stretching direction was observed only for isolated CNTs but not for the clusters.

The extensional rheology for both treated and untreated CNT suspensions has been studied by Ma et al. [115] using a filament stretching method. A liquid sample was first loaded between two cylindrical endplates forming a liquid bridge. Both endplates were then moved apart (at $t=0$ ) by a known distance and at a constant rate. After the initial stretching, the filament thinning process was captured using a high-speed camera. Figure 10 is a sequence of images showing the extensional deformation for the filaments of (a) epoxy, (b) $0.02 \mathrm{wt} \%$ untreated CNTs and (c) $0.3 \mathrm{wt} \%$ treated CNTs suspended in the epoxy.

The treated and untreated suspensions were prepared at different CNT concentrations to match their

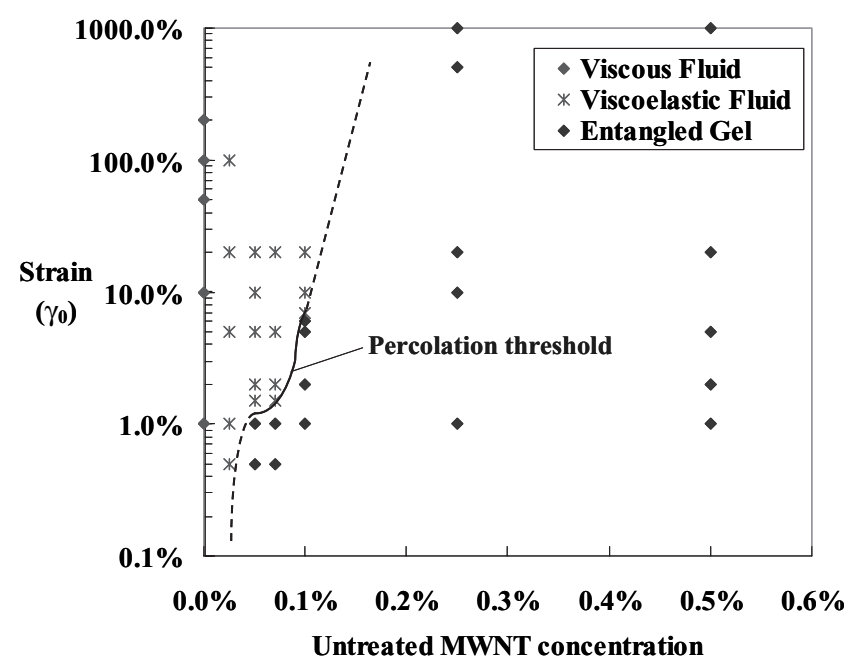

Fig. 9 Phase diagram for untreated MWNT suspensions: different concentrations of the suspensions were subjected to different magnitudes of strain and results similar to Fig. 8 were then summarized to construct the phase diagram. Depending on the strain magnitude and concentration level, untreated MWNT suspensions finally dissipated as a viscoelastic fluid or an entangled gel, whereas the epoxy matrix demonstrated a viscous fluid dissipation for all strain levels applied [59] 


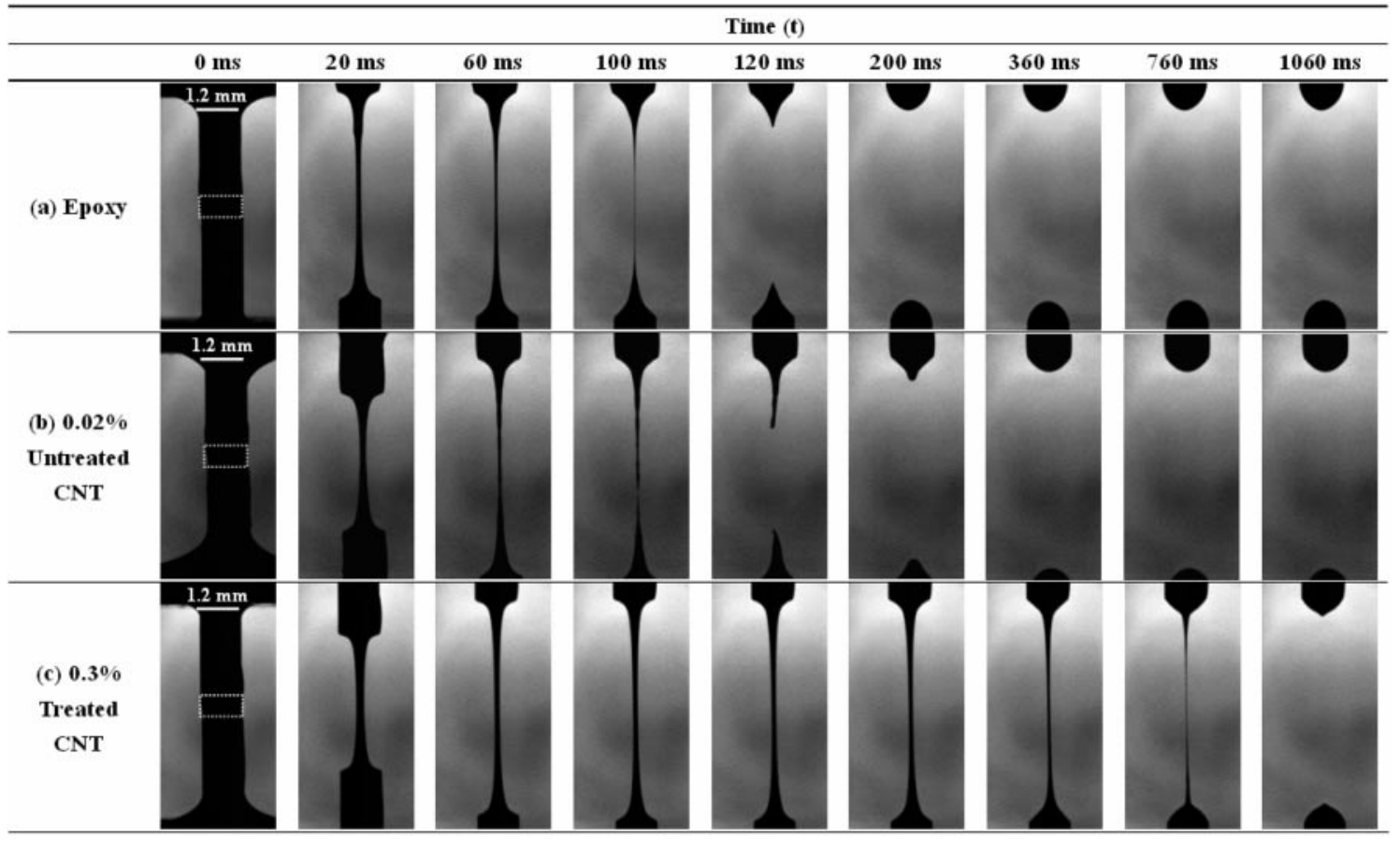

Fig. 10 Sequence of images showing the extensional deformation for the filaments of (a) epoxy, (b) $0.02 \mathrm{wt} \%$ untreated CNT suspension, and (c) $0.3 \mathrm{wt} \%$ treated CNT suspension. Stretching rate $=100 \mathrm{~mm} / \mathrm{s}$; endplate displacements $=3 \mathrm{~mm}$ from starting positions. The initial location of liquid sample at $t=0 \mathrm{~ms}$ highlighted using dotted lines [115]

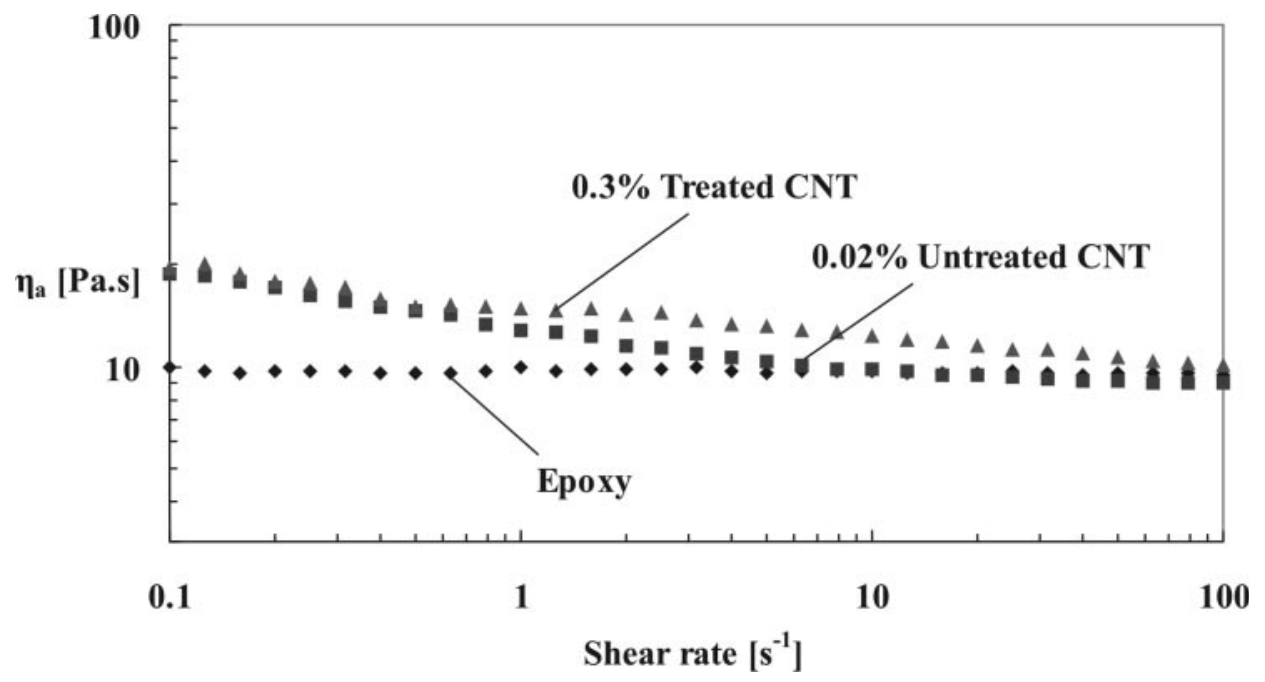

Fig. 11 Apparent steady shear viscosities, $\eta_{\mathrm{a}}$, of epoxy, untreated and treated CNT suspensions. The low-shear-rate viscosities of the CNT suspensions were matched by adjusting the concentrations [115]

low-shear viscosity. Although both samples showed similar response in steady shear, the addition of chemically treated CNT (Fig. 11) was found to increase the filament breakup time significantly in extensional flow (Fig. 12). This was believed to be a result of CNT orientation along the filament stretching direction as a similar effect was reported in the case of glass fibre suspensions [116]. The degree of alignment was subsequently estimated and it was calculated that about 99 per cent of the $0.3 \mathrm{wt} \%$ treated CNTs were oriented in the filament stretching direction after stretching with a strain input of 5 . In contrast to the smooth filaments formed by chemically treated CNT suspensions, filaments formed with untreated CNT suspensions were found to behave in a non-uniform way with local fluctuation in filament 
diameter (as shown in Fig. 13(b)), making the extensional viscosity calculations unreliable [115]. The irregularity of the untreated CNT filaments was consistent with optical images, where CNT aggregates were observed (Fig. 13(e)).

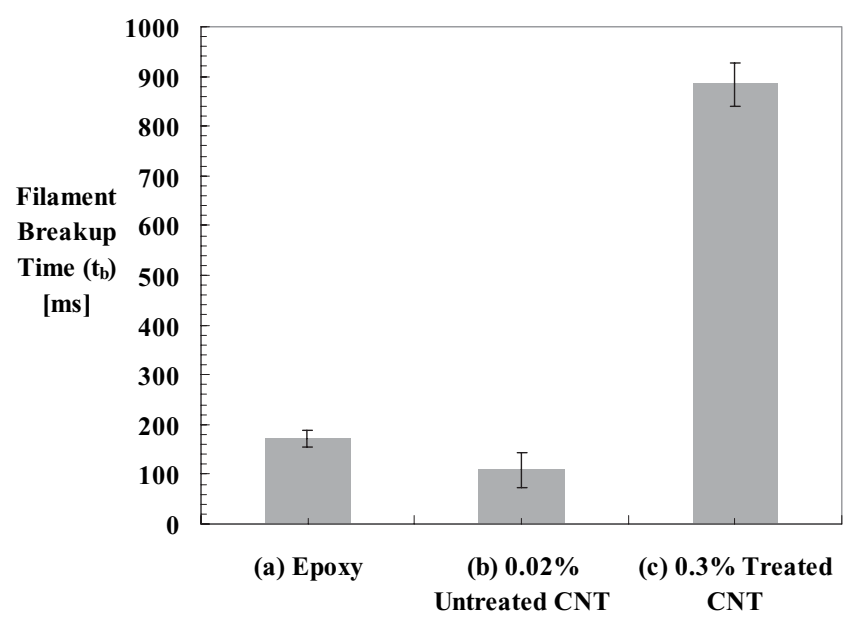

Fig. 12 Filament breakup time, $t_{\mathrm{b}}$, for (a) epoxy, (b) $0.02 \mathrm{wt} \%$ untreated CNT suspension, and (c) $0.3 \mathrm{wt} \%$ treated CNT suspension. Stretching rate $=100 \mathrm{~mm} / \mathrm{s}$; endplate displacements $=3 \mathrm{~mm}$ from starting positions [115]

\section{KEY PARAMETERS THAT AFFECT THE RHEOLOGY OF CNT SUSPENSIONS}

\subsection{Dispersion state}

A number of rheological studies have shown that the rheology of CNT suspensions is closely linked to their dispersion state $[\mathbf{3 1}, 34,38,47]$. Huang and coworkers [38] further proposed that rheology can be used as a means to assess the state of dispersion. The authors prepared CNT samples with different mixing times and characterized the LVE response. They observed that there existed a certain critical dispersion time beyond which a stable rheology was established. A mixing time of at least several hours was required to achieve a rheologically stable dispersion for their highly entangled CNTs and shear mixer.

Du et al. [31] prepared CNT/PMMA samples with different dispersion states and observed that the better dispersed samples showed a higher storage modulus $G^{\prime}$ and a $G^{\prime}$ plateau at low frequency. Similar findings were reported by Fan and Advani [47] for CNTs dispersed in epoxy. However, some authors such as Song and Youn [34] and Huang et al. [38] found the opposite to be true. Fan and Advani [47] attempted to reconcile the discrepancy by suggesting that different microstructures were present in $\left(\mathrm{t}-\mathrm{t}_{\mathrm{s}}=156 \mathrm{~ms}\right)$
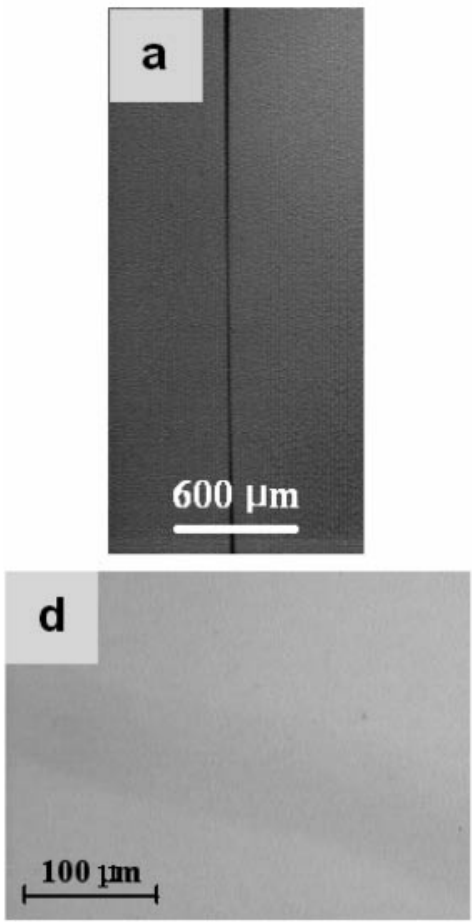
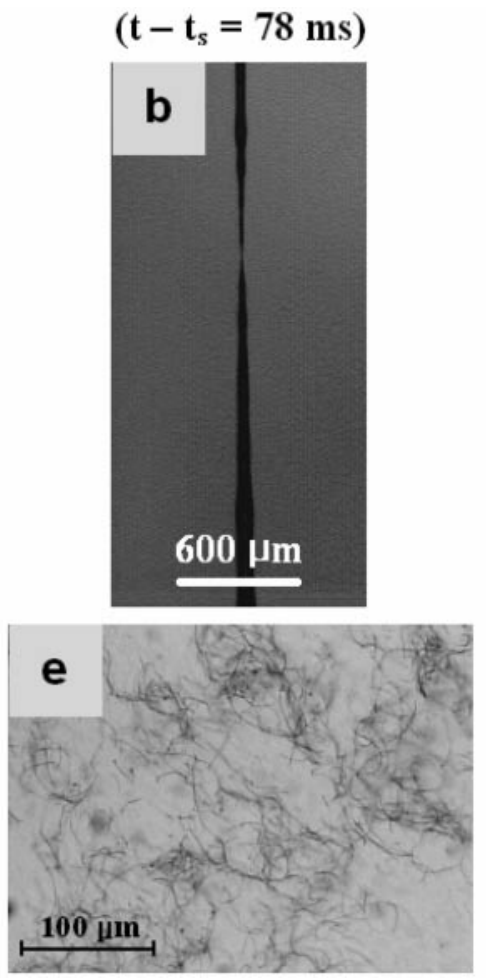
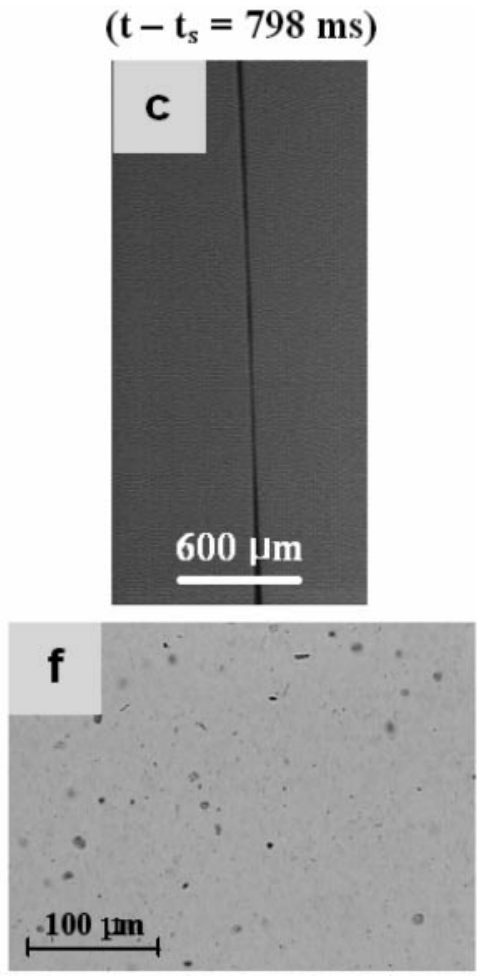

Fig. 13 The filament profile before breakage for (a) epoxy, (b) $0.02 \mathrm{wt} \%$ untreated CNT, and (c) $0.3 \mathrm{wt} \%$ treated CNT suspensions; (d)-(f) are corresponding optical micrographs captured using the Cambridge Shear System (CSS450, Linkam Scientific Instruments) with an optical depth of $130 \mu \mathrm{m} . t_{\mathrm{s}}$ is the time at which the endplates stopped [115] 
these studies. In essence, all the experimental findings suggested that the rheological response of CNT suspensions was affected by the dispersion state, but judging the state of dispersion merely on the presence (or absence) of a $G^{\prime}$ plateau could be misleading. Rheologically, a low-frequency plateau of $G^{\prime}$ suggested the presence of a physical network but it did not necessarily imply that the dispersion state is good because a poorly dispersed sample containing interconnected CNT aggregates could also result in a low-frequency $G^{\prime}$ plateau.

More recently, more than one research group [117, 118] reported that high-temperature treatment, also known as 'annealing', could increase the conductivity of extruded CNT composites. It was believed that annealing had promoted aggregation and thus the formation of a percolating network within the composite. In other words, a certain degree of aggregation can be advantageous for producing conductive CNTbased composite materials, provided that optical transparency is not a major concern.

\subsection{Suspending medium}

In many cases, a suspending medium is chosen based on the final application but this choice could influence the rheology of the CNT composite in a number of ways. Depending on the chemical composition and base viscosity, different suspending media have different abilities to wet and disperse CNTs [57, 119]. Martin and co-workers [120] reported that CNTs can be dispersed favourably in epoxy resin, but the addition of epoxy hardener to CNT/ epoxy resin mixtures resulted in the re-aggregation of CNTs. They suggested that as-produced CNTs were negatively charged as in the case of carbon black and that the addition of hardener decreased the ionic strength of the suspending medium, creating an environment that favours aggregation. The choice of suspending medium could influence the state of dispersion, which in turn can modify the rheology.

For non-Newtonian suspending media, Hobbie et al. [27] observed that at high shear rates, CNTs suspended within a weakly elastic fluid tended to align in the vorticity direction whereas those suspended within a highly elastic fluid preferentially oriented in the flow direction. Their experimental observations are consistent with previous experimental and theoretical studies for fibre suspensions [121-124].

\subsection{Chemical treatment}

Ma [59] studied the effect of chemical treatment on the rheological behaviour of CNTs suspended within a Newtonian epoxy medium (without hardener) (Figs 14-16). It was found that the extent of the shearthinning effect, as described in section 5.1, depended strongly on the type of CNT. Untreated CNT suspensions with optically resolvable aggregate structures tended to exhibit a more significant shearthinning effect compared with the treated CNT suspension showing little optical microstructure (Fig. 14). In terms of LVE, both treated and untreated CNTs increased the elasticity of the system, but the treated CNT suspension with little optical microstructure showed more delicate elasticity (Fig. 15). Similar experiments were performed by Fan and Advani [47] who compared untreated MWNTs with acid-treated MWNTs suspended in epoxy. TEM images of the two suspensions showed that the aspect ratio of the treated CNTs was much smaller than that of the untreated CNTs and this was used to explain the more pronounced increase in $G^{\prime}$ and $G^{\prime \prime}$ seen with the untreated CNTs (see section 6.4).

\subsection{Aspect ratio}

Fan and Advani [47] reported that suspensions containing CNTs having high aspect ratio $(\sim 500)$ showed larger $G^{\prime}$ and $G^{\prime \prime}$ values than those with low aspect ratio $(\sim 25-50)$. The authors explained that it was easier for CNTs with a higher aspect ratio to form an interconnected network within the suspension. In a more recent study by Rahatekar et al. [125] where the CNTs were shortened using sonication, the authors reported that short CNTs tended to behave as long CNTs at high concentrations, but short CNTs could be processed more easily as the network formed tended to have a lower yield stress. The studies by Fan and Advani [47] and Hobbie [126] were of the few studies covering the effect of aspect ratio systematically, but it should be noted that in the study of Fan and Advani [47], the CNTs were shortened using chemical means which had probably also modified the surface chemistry and thus the interaction potential of the CNTs.

\subsection{Concentration}

The effect of concentration on rheological response was investigated in many of the rheological studies listed in Table 2. The influence of CNTs on the steady shear and LVE rheology generally became more pronounced for increasing CNT concentration. Intuitively, as the CNT concentration increases, the inter-CNT spacing decreases and there exists a critical concentration above which a network is formed and a dramatic increase in viscosity is observed. Of particular interest is the lower concentration limit for the network formation, also known as the rigidity or rheological percolation threshold $[101,106]$. Given the high aspect ratio of CNTs, percolation for CNT systems was usually achieved at relatively low concentration, typically smaller than $1 \mathrm{wt} \%$, as summarized in Table 3. 


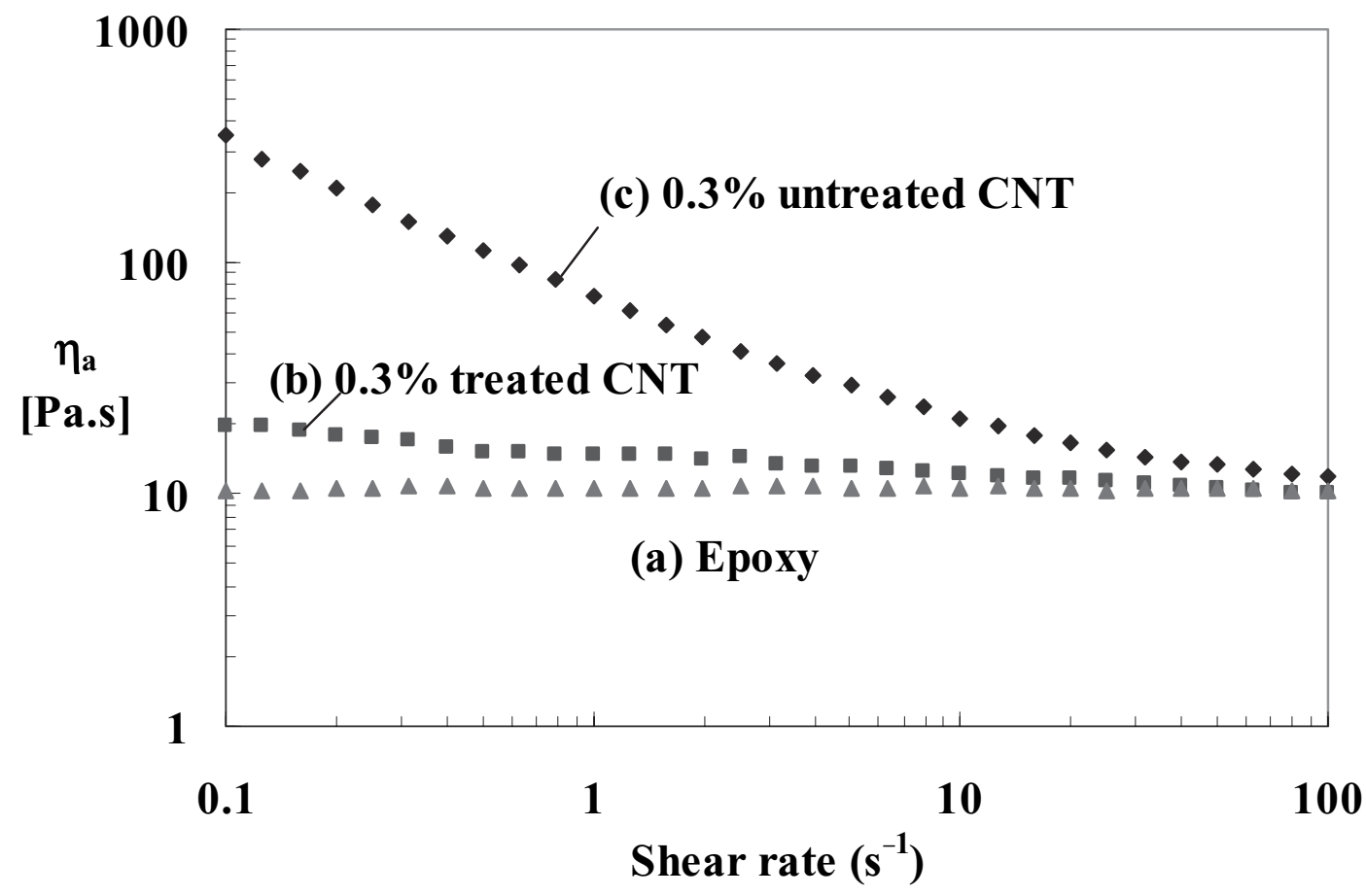

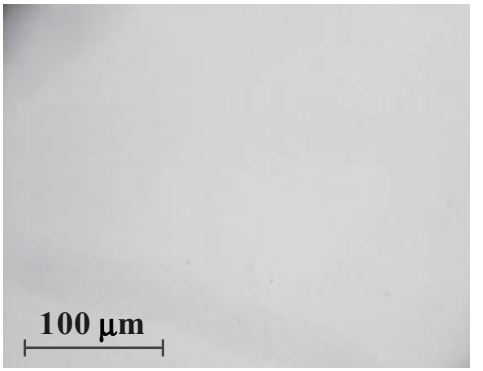

(a) Epoxy

$\left(\right.$ Shear rate $\left.=0 \mathrm{~s}^{-1}\right)$

\section{Optical Micrographs}

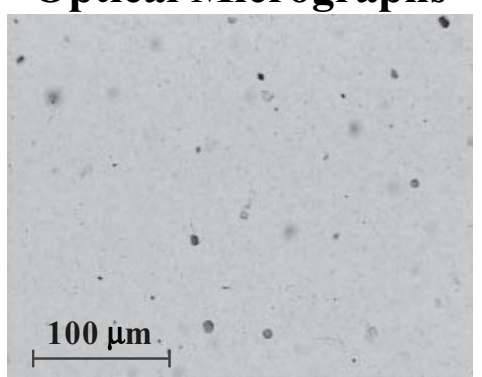

(b) $0.3 \%$ treated CNT (Shear rate $=0 \mathrm{~s}^{-1}$ )

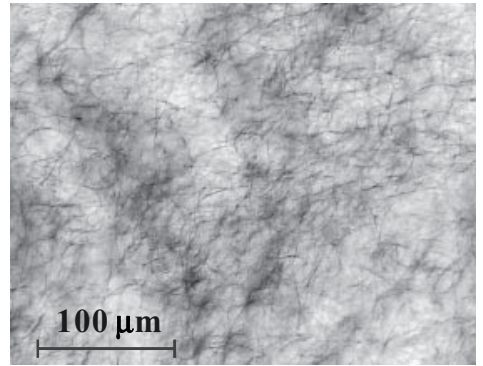

(c) $0.3 \%$ untreated CNT $\left(\right.$ Shear rate $=0 \mathrm{~s}^{-1}$ )

Fig. 14 Apparent steady shear viscosity, $\eta_{\mathrm{a}}$, of (a) epoxy, (b) $0.3 \mathrm{wt} \%$ treated CNT, and (c) $0.3 \mathrm{wt} \%$ untreated CNT with matching quiescent optical micrographs (optical depth $=130 \mu \mathrm{m}$ )
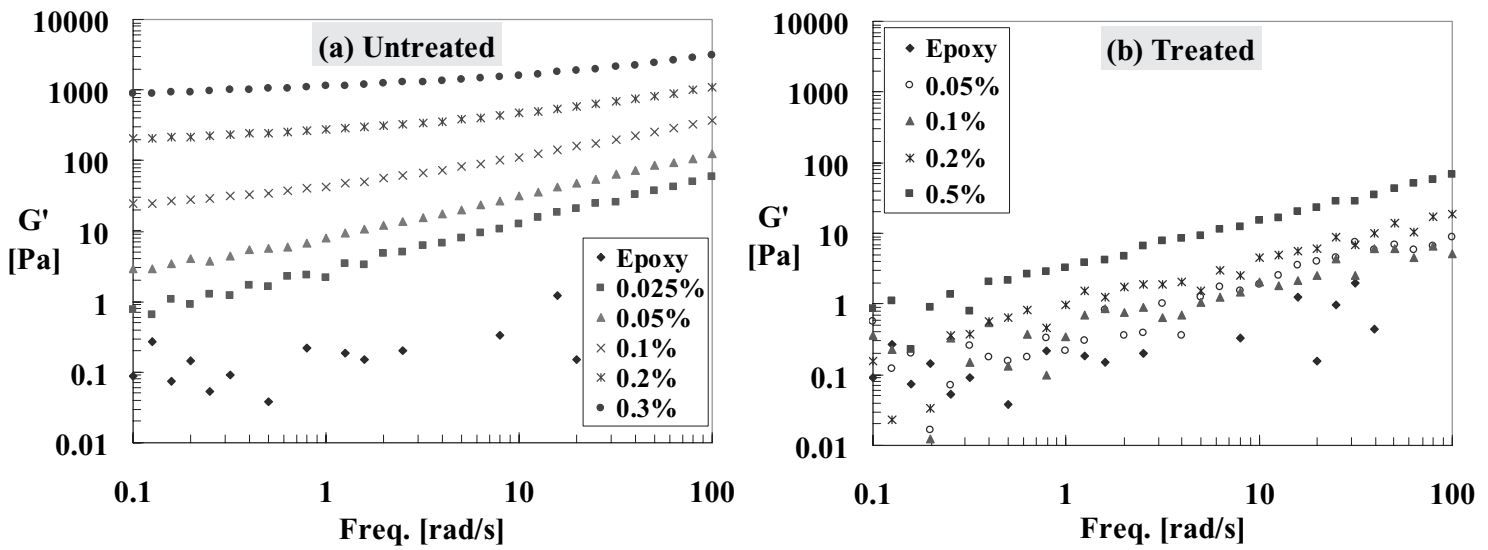

Fig. 15 Storage modulus, $G^{\prime}$, data for different concentrations (wt\%) of (a) untreated SWNT and (b) treated SWNT suspensions. Strain $=1$ per cent; temperature $=25^{\circ} \mathrm{C}[\mathbf{5 9}]$ 


\section{MODELLING}

As discussed in section 5.1, numerous attempts have been made to fit constitutive equations to the rheological data obtained from CNT composites. These include the Cross equation [25], the Carreau equation [41], the Krieger-Dougherty equation [35], the Bingham model, and the Herschel-Buckley model [42]. Nevertheless, some authors have also tried to formulate models based on a physical understanding of the interactions between CNTs and matrix and the behaviour of CNTs under shear.

A simple model was devised by Hough et al. [33] to explain the strain-induced fluidization of aqueous SWNT suspensions. Their LVE data gave a plateau in $G^{\prime}$ at low frequencies, and from this it was postulated that there existed a CNT network composed of freely jointed associating rods within the matrix. At the onset of fluidization $\left(G^{\prime \prime}>G^{\prime}\right)$, this network is destroyed because the CNTs have rotated through angles with arc length greater than the bond interaction range. By considering the relationship between the maximum rotation angle, the mesh size of the network, and the nanotube length, Hough et al. [33] were able to derive a relationship between the

Table 3 Some reported values for the rheological percolation threshold of CNT suspensions

\begin{tabular}{llll}
\hline Nanotube & $\begin{array}{l}\text { Suspending } \\
\text { medium }\end{array}$ & $\begin{array}{l}\text { Rheological } \\
\text { percolation } \\
\text { threshold (wt\%) }\end{array}$ & Reference \\
\hline SWNT & PMMA & 0.12 & {$[\mathbf{3 1}]$} \\
MWNT & Epoxy & 0.1 & {$[\mathbf{2 5}]$} \\
& PC & $0.25-0.5$ & {$[\mathbf{1 2 7}]$} \\
& Water & $0.5-0.7$ (vol\%) & {$[\mathbf{8 4}]$} \\
\hline
\end{tabular}

CNT, carbon nanotube; SWNT, single-walled carbon nanotube; MWNT, multi-walled carbon nanotube; PMMA, poly(methyl methacrylate); PC, polycarbonate. macroscopic yield strain and CNT volume fraction which agreed with their experimental data.

The authors of the present paper modelled both aggregating and non-aggregating CNT suspensions in greater detail using a stress-microstructure approach (see references [60] and [94] for detailed model formulation). In both studies, the CNTs were suspended within a Newtonian epoxy matrix. A Fokker-Planck (FP)-based orientation model was found to describe reasonably well the shear-thinning behaviour observed for CNTs which had been chemically treated such that they would not aggregate [94]. Two adjustable parameters, $N_{\mathrm{p}}$ and $D_{\mathrm{r}}$, were used in the model fitting of non-aggregating CNT suspensions (as shown in Fig. 17(a)). $N_{\mathrm{p}}$ is a concentrationdependent parameter which scaled linearly with CNT concentration, whereas $D_{\mathrm{r}}$ is the rotary diffusion coefficient, describing the rate of misalignment from the flow direction due to randomizing events. As discussed in reference [94], the best $D_{\mathrm{r}}$ value determined from fitting the model to experimental data of semi-dilute CNT suspensions was found to be higher than the theoretical value predicted by the DoiEdwards [128] theory for dilute suspensions. The higher value of $D_{\mathrm{r}}$ could be explained by additional interparticle interaction in the case of semi-dilute suspensions [129]. The success of the model suggested that the non-aggregating CNTs behave essentially as short rigid fibres and the corresponding shear-thinning behaviour was due to the progressive alignment of CNT in the shear direction. In reference [94], the degree of alignment was quantified using a scalar order parameter $S$ commonly used in liquid crystal (polymer) studies $[\mathbf{6 0}, 130] . S$ varies between 0 and 1 and the larger the magnitude of $S$, the higher the degree of alignment. As shown in Fig. 17(c), the FP-based orientation model predicted a high level of alignment where $S=0.92$. Despite the success in modelling the steady shear behaviour of
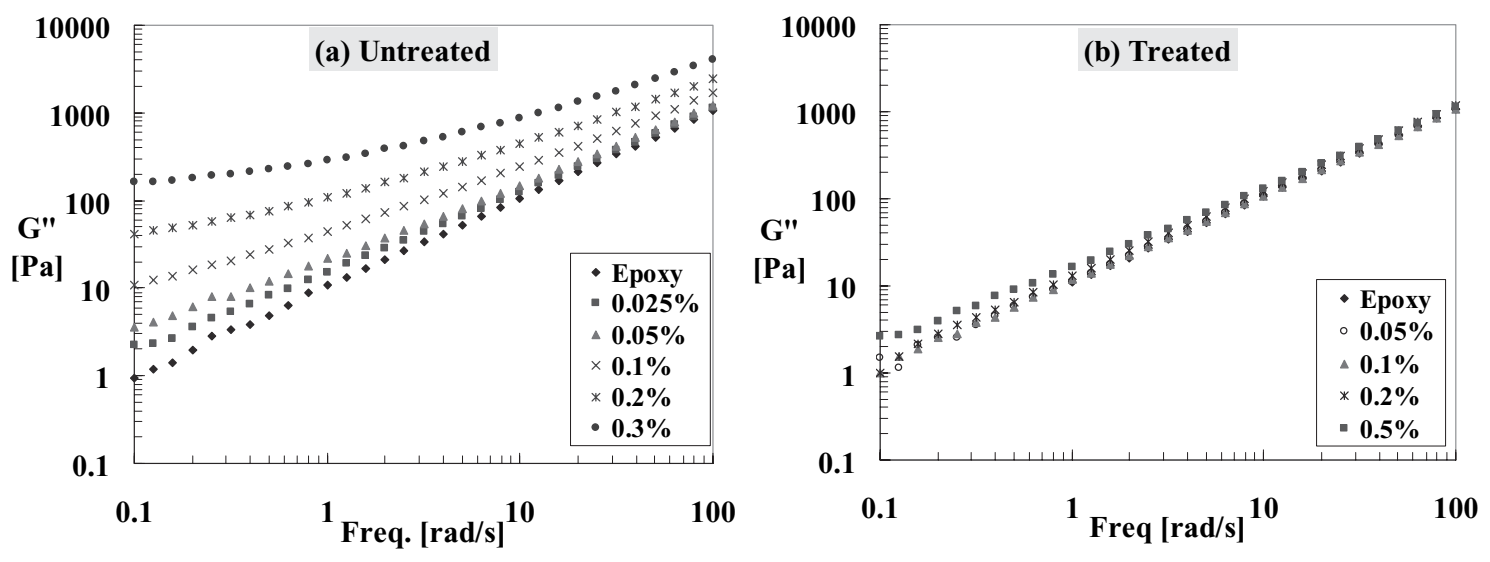

Fig. 16 Loss modulus, $G^{\prime \prime}$, data for different concentrations (wt\%) of (a) untreated SWNT and (b) treated SWNT suspensions. Strain $=1$ per cent; temperature $=25^{\circ} \mathrm{C}[\mathbf{5 9}]$ 

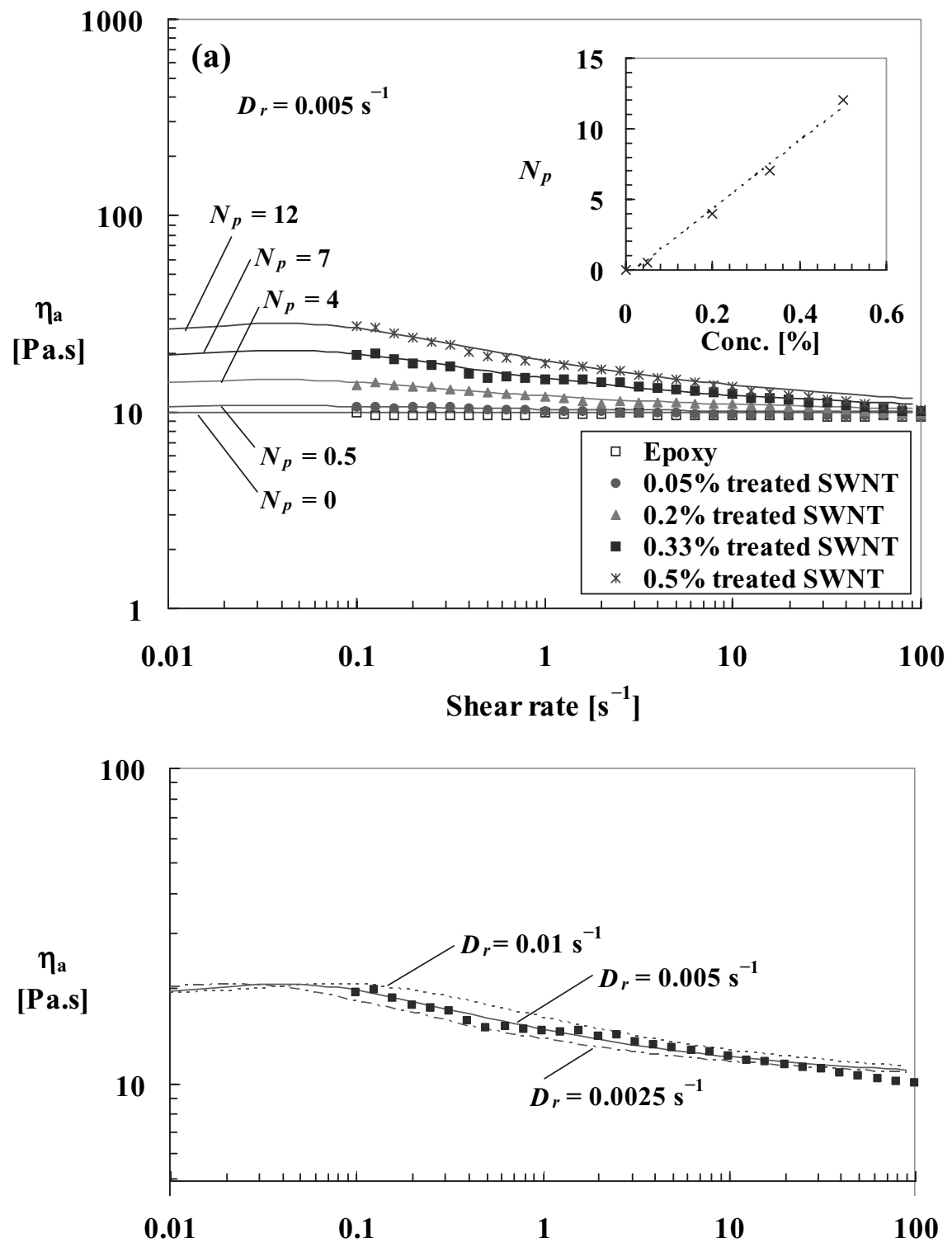

Shear rate $\left[\mathrm{s}^{-1}\right]$

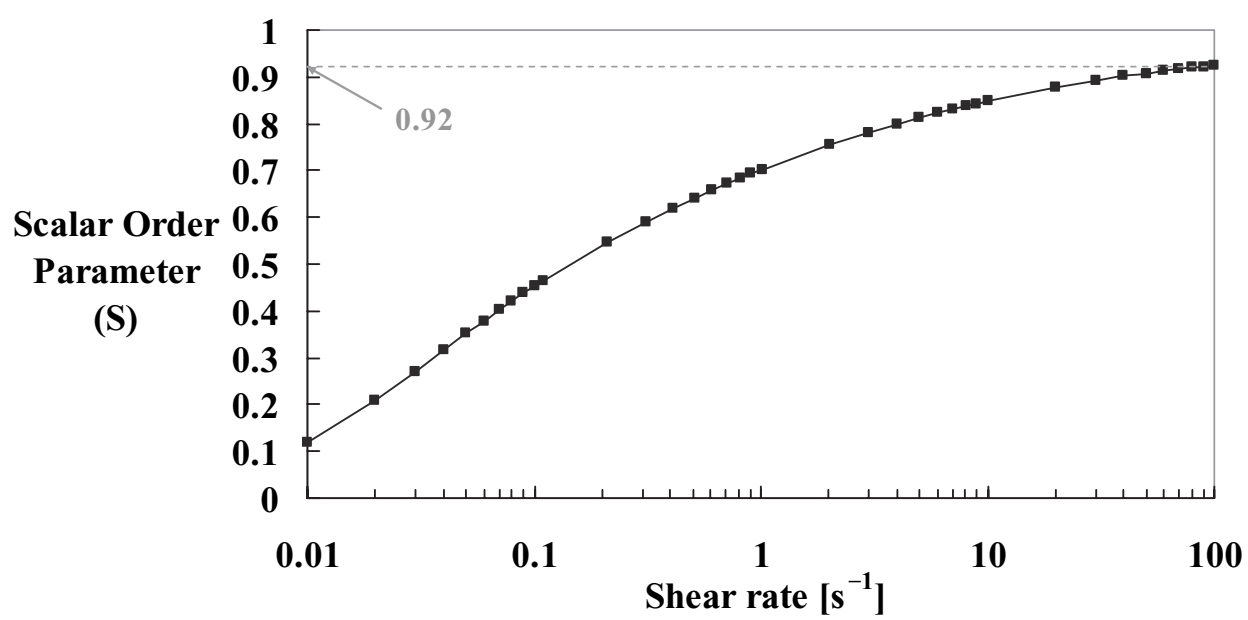

Fig. 17 (a) Orientation model fittings for $0.05 w t \%, 0.2 w t \%, 0.33 w t \%$, and $0.5 w t \%$ treated SWNTs suspended in epoxy resin. $N_{\mathrm{p}}$ and $D_{\mathrm{r}}$ are the fitting parameters. (b) Sensitivity of the model to $D_{\mathrm{r}}$ given the experimental data of $0.3 \mathrm{wt} \%$ treated CNT suspension and $N_{\mathrm{p}}=7$. (c) Scalar order parameter, $S$, as a function of shear rate, where $0 \leqslant S \leqslant 1$ 
non-aggregating CNT suspensions using an FP-based orientation model, modelling the LVE data was found to be more challenging. Experimental LVE data of the treated CNT suspensions were fitted using the FP orientation model with an 'effective diffusion coefficient' term and a purely empirical relationship was subsequently identified, where the 'effective diffusion term' was found to scale with the square root of the frequency. The phenomenological relationship could possibly originate from the interaction between weakly interconnected CNTs where each CNT is subjected to an extra force (that scales with the square root of frequency). In the literature, a number of authors $[\mathbf{1 3 1}, \mathbf{1 3 2}]$ have proposed that particle-particle interactions could result in elasticity. In the modelling of polymers filled with interacting particles, Leonov [131] considered the release of 'elastic energy' during the rupturing of flocs of particles; whereas Dinsmore et al. [132] suggested that the interactions between colloidal particles could be represented by conceptual springs.

Compared with non-aggregating CNT suspensions, CNT suspensions containing optically resolvable CNT aggregates tended to demonstrate a stronger shear-thinning effect, where the orientation effect alone was found to be insufficient in describing the experimental data [60]. Inspired by earlier work on associative polymer modelling [133-135], a more sophisticated model named the 'aggregation/ orientation' model was formulated and verified against experimental results (as shown in Fig. 18) [60]. The new model considered that a hierarchy of CNT aggregate structures was present in the suspension and the tendency for CNTs to align in the shear direction depended on the flow conditions as well as the entanglement state of CNTs. Similar to the FP-based orientation modelling, $N_{\mathrm{p}}$ and $D_{\mathrm{r}}$ were model fitting parameters and only one additional fitting parameter, $\alpha$, was introduced in the simplest form of the aggregation/orientation model. $\alpha$ represents the relative rate of disaggregation to aggregation and was found to decrease for increasing CNT concentration, implying that it would be more difficult to disentangle CNT at high loadings (see reference [60] for details). The aggregation/orientation modelling results were found to be consistent with experimental observations that CNT tended to aggregate at low shear rates but became disentangled at high shear rates (section 4.2). Figure 19 shows the CNT (aggregate) population distributions simulated for four different concentrations of CNT suspensions sheared at two different rates. The $x$-axis, $n$, describes (a) $0.25 \% \mathrm{CNT}$

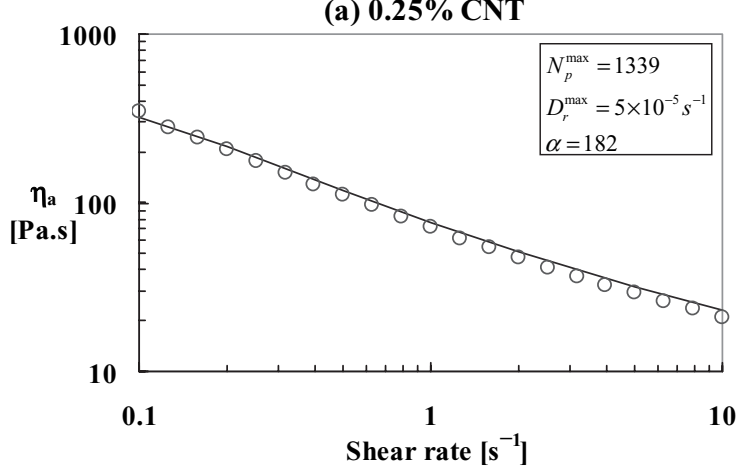

(c) $0.05 \% \mathrm{CNT}$

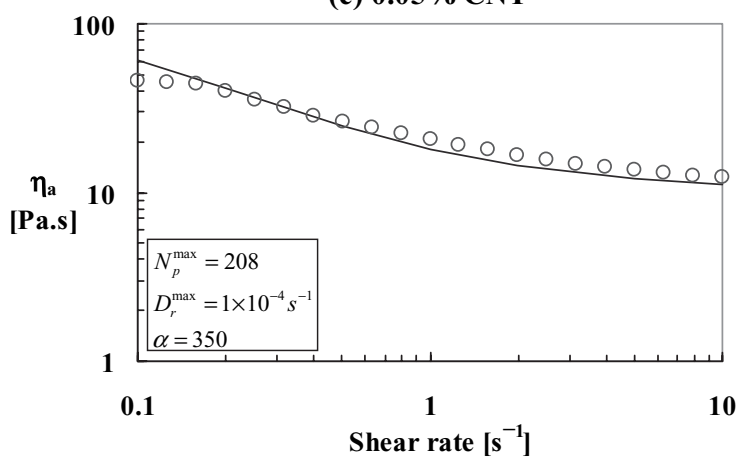

(b) $0.1 \% \mathrm{CNT}$

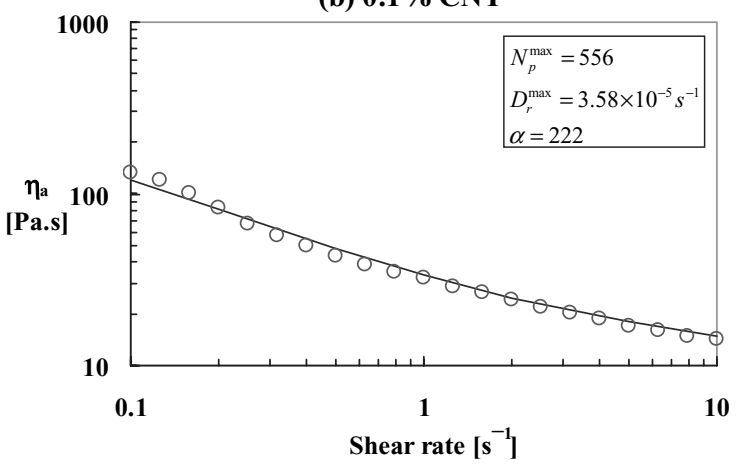

(d) $0.025 \%$ CNT

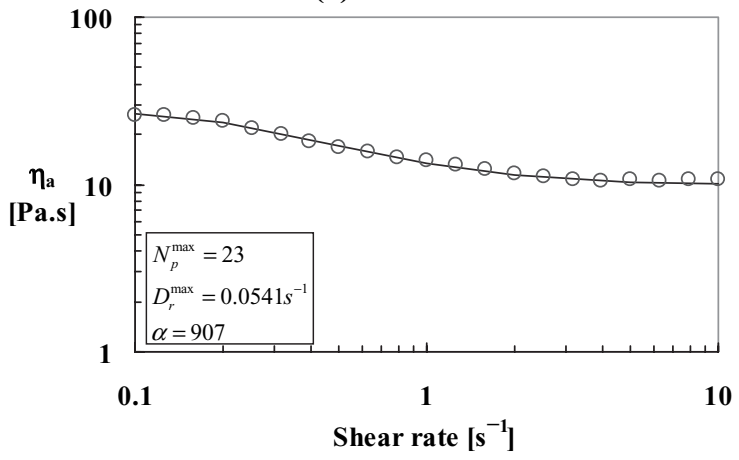

Fig. 18 Aggregation/orientation model fitting to experimental $\eta_{\mathrm{a}}$ data at different CNT concentration levels: (a) $0.25 \mathrm{wt} \%$, (b) $0.1 \mathrm{wt} \%$, (c) $0.05 \mathrm{wt} \%$, and (d) $0.025 \mathrm{wt} \%$. Experimental data are represented by unfilled circles ( $\circ$ ) and the best fit values of $N_{\mathrm{p}}^{\max }, D_{\mathrm{r}}^{\max }$, and $\alpha$ were identified using the least-squares method (see reference [59]) 
the state of CNT entanglement, with $n=0$ referring to nanotubes free from entanglement and $n=1$ representing CNT entangled in the highest possible state. As shown in Fig. 19, the population distribution skewed towards $n=1$ (CNTs tended to aggregate) at low shear $\left(0.1 \mathrm{~s}^{-1}\right)$ but skewed towards $n=0$ (CNTs became less entangled) at high shear. It is also clear from the figure that the population distribution tended to skew (more) towards $n=1$ for increasing CNT concentration, meaning that high concentration CNT samples had a higher tendency to aggregate.
Similar FP-based approaches have been used by Song and Youn [136] and Qiu et al. [137], but these authors modelled CNTs as finitely extensible nonlinear elastic (FENE) dumbbells, instead of inextensible rigid rods. A FENE dumbbell can be visualized as consisting of two beads joined with a mass-less non-linear spring. The beads symbolize the interaction point between the CNT and the solvent whereas the spring corresponds to the stretching of CNTs. Aggregated CNTs are modelled using a FENE dumbbell with lower mobility (lower translational

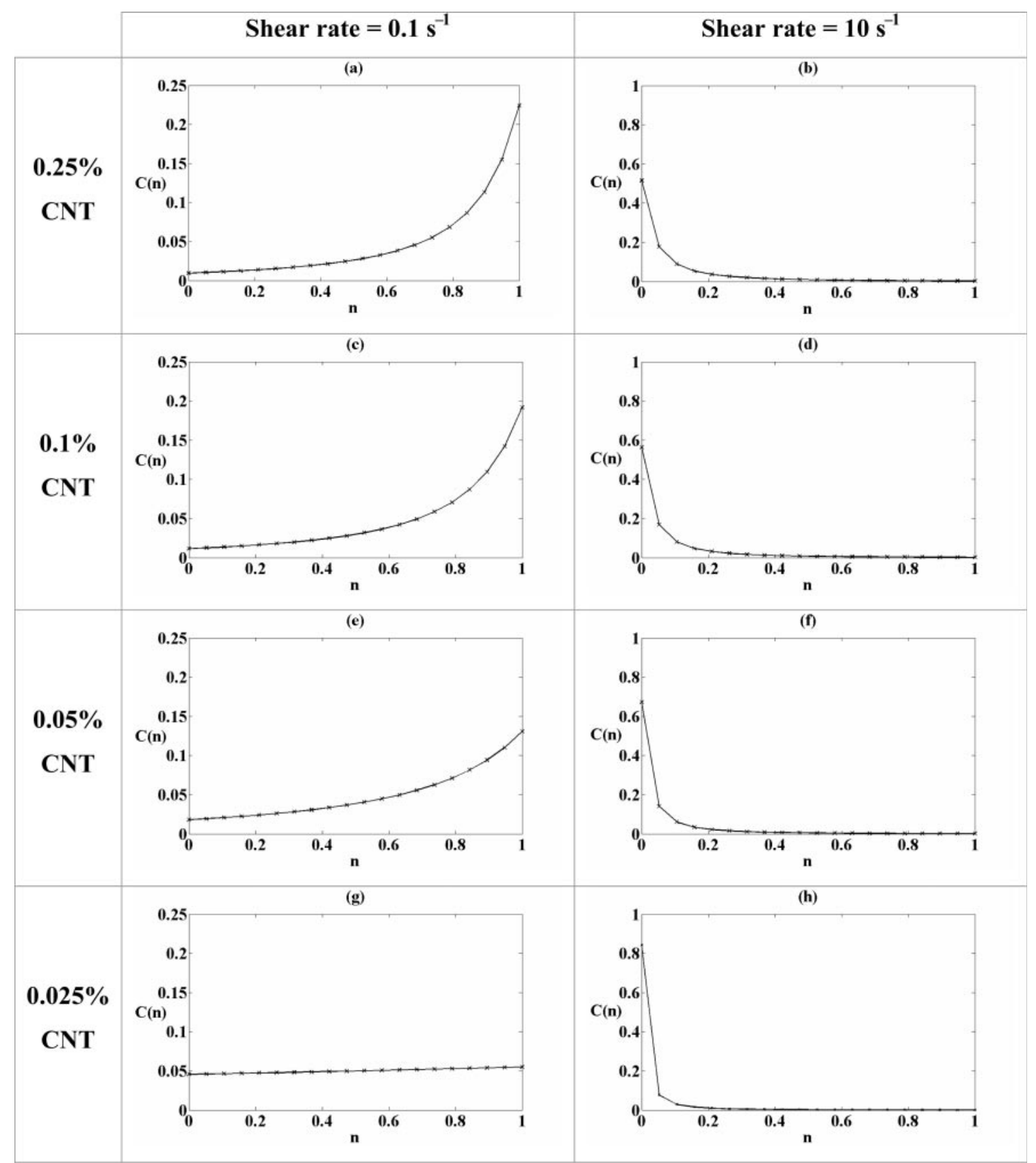

Fig. 19 Aggregation/orientation model: the effect of CNT concentration (wt\%) and magnitude of shear rate on the CNT population distributions, $C(n)$. This figure shows that CNT tended to aggregate (population distribution skews towards $n=1$ ) at high concentration and low shear conditions 
diffusivity) and free CNTs are modelled using a FENE dumbbell with higher mobility (higher translational diffusivity). Unlike the work of Ma et al. [115], the models used by Song and Youn [136] and Qiu et al. [137] considered only two populations, as in the original work of Vaccaro and Marrucci [133] for associative polymers. The model showed good quantitative agreement with experimental data for steady shear flows but failed to describe the experimental data quantitatively for oscillatory shear flows.

It is worth noting that although the models reported in references $[\mathbf{6 0}]$ and $[\mathbf{9 4}]$ were originally developed for describing simple rheometric flows, these models can be combined with other modelling methods to allow for the simulation of more complex processing flows. Cueto et al. [138], for example, modelled the spin-coating process involving nonaggregating CNTs by coupling the FP-based orientation model with the natural element method, which described the thinning process of the CNT suspension on a rotating substrate. The combined model provided theoretical predictions for the velocity field as well as the CNT orientation field as the CNT suspension was spun into a film.

Despite recent advances in the modelling of CNT, there are a number of open questions yet to be answered. First, although the steady shear-thinning behaviour of both treated and untreated CNT suspensions was modelled, there has been little success in modelling the evolution of storage modulus $G^{\prime}$ as a function of frequency. Experimental LVE data of treated CNT suspensions could be modelled using an FP-based orientation model, but the model also involved the use of a purely empirical diffusion coefficient, of which the physical origin has not been completely understood. Second, in addition to particle-particle interaction, CNT bending would store up elastic energy and is therefore a possible origin for the elasticity of treated nanofibre/nanotube systems [31]. For future work, it is of interest to model the bending of CNT using a kinetic theory approach, and evaluate its potential contribution towards elasticity observed experimentally. Third, in the case of aggregating CNT suspensions, CNTs in different entanglement states were observed to have different tendencies to align in flows as reported by Hobbie et al. [26, 27]. To reflect this experimental observation, it would be useful to model CNTs according to their entanglement states. To summarize, actual CNT systems may involve physical mechanisms that are more complex than what existing models assume and the construction of a universal and self-consistent model for different types of CNTs and flow conditions still remains a challenging task.

\section{CHALLENGES AND CONCLUSIONS}

Based on experimental findings and modelling efforts over the last decade, a scientific picture is emerging but there are key challenges yet to be overcome. In terms of fundamental understanding, the origin for elasticity observed in CNT systems still remains unclear. As pointed out in reference [60], the classical non-Brownian fibre suspension model predicted no elasticity [139] whereas elastic dumbbell models used by Qiu et al. [137] and Song and Youn [136] predicted single-mode Maxwell-type elasticity. Experimentally, the treated CNT suspensions showed mild elasticity that could not be described by the classical non-Brownian fibre model or the elastic dumbbell model. Different authors have put forward different hypotheses for explaining the elasticity [33, 114] and there is no consensus over the physical origin for the elasticity observed in the treated CNT suspensions.

Although some progress has been made; the microstructure and rheology of CNT suspensions still raise many unresolved issues. Effective methods of dispersion in ordinary fluids without significantly reducing nanotube length have not yet been fully developed and the intrinsic affinity that nanotubes have to each other means that the prevention of CNT aggregation remains challenging. While it is possible to develop microstructural models for CNT suspension rheology, all models will depend on states of aggregation and CNT ordering. This presents difficulties as both aspects are difficult to access at length scales below optical resolution. In principle, TEM, scanning electron microscopy, atomic force microscopy, Raman, X-ray and other techniques can provide valuable information; however, with only a few exceptions, it has so far proved consistently difficult to obtain reliable and conclusive nanometre and submicrometre information for CNT suspensions. Until this information is obtained the absolute validity of much of the rheological modelling that has been carried out remains uncertain.

It is clear that the science of CNT suspensions is both challenging and interesting. The materials fall between polymer chain and fibre suspension behaviour, with the added complexity associated with aggregate formation and the richness of different CNT variants. The future development of the science of CNTs will, however, very much depend on future applications and this in turn will depend on costeffective manufacture of CNTs and their dispersion, together with environmental acceptance. If CNTs are found to be dangerous to human health, then their scope for large-scale application similar to polymers and fibre composites will be greatly reduced. At present electrical conductivity enhancement of thermoplastic and thermoset systems looks the most 
promising large-scale application area, with the possible added bonus for some systems where optical transparency and conductivity can occur together. On the other hand, the application potential for CNT suspensions is very broad and many barely unexplored opportunities still exist to develop new classes of materials with unexpected properties and exciting new uses.

\section{ACKNOWLEDGEMENTS}

A.W.K. Ma would like to thank the Croucher Foundation for the award of a PhD Scholarship to Cambridge University and K.M. Yearsley would like to acknowledge EPSRC for a Doctoral Training Scholarship.

\section{(c) Authors 2009}

\section{REFERENCES}

1 Ebbesen, T. W. and Ajayan, P. M. Large-scale synthesis of carbon nanotubes. Nature, 1992, 358, 220-222.

2 José-Yacamán, M., Miki-Yoshida, M., Rendón, L., and Santiesteban, J. G. Catalytic growth of carbon microtubules with fullerene structure. Appl. Phys. Lett., 1993, 62, 657-659.

3 Dai, H., Rinzler, A. G., Nikolaev, P., Thess, A., Colbert, D. T., and Smalley, R. E. Single-wall nanotubes produced by metal-catalyzed disproportionation of carbon monoxide. Chem. Phys. Lett., 1996, 260, 471-475.

4 Singh, C., Shaffer, M. S. P., and Windle, A. H. Production of controlled architectures of aligned carbon nanotubes by an injection chemical vapour deposition method. Carbon, 2003, 41, 359-368.

5 Guo, T., Nikolaev, P., Rinzler, A. G., Tománek, D., Colbert, D. T., and Smalley, R. E. Self-assembly of tubular fullerenes. J. Phys. Chem., 1995, 99, 1069410697.

6 Kingston, C. T. and Simard, B. Fabrication of carbon nanotubes. Anal. Lett., 2003, 36, 3119-3145.

7 Liu, J., Fan, S., and Dai, H. Recent advances in methods of forming carbon nanotubes. MRS Bull., 2004, April, 244-250.

8 Iijima, S. Helical microtubules of graphitic carbon. Nature, 1991, 354, 56-58.

9 Saito, S. Carbon nanotubes for next-generation electronics devices. Science, 1997, 278, 77-78.

10 Calvert, P. Nanotube composites: a recipe for strength. Nature, 1999, 399, 210-211.

11 Andrews, R., Jacques, D., Rao, A. M., Rantell, T., Derbyshire, F., Chen, Y., Chen, J., and Haddon, R. C. Nanotube composite carbon fibers. Appl. Phys. Lett., 1999, 75, 1329-1331.

12 de Heer, W. A. Nanotubes and the pursuit of applications. MRS Bull., 2004, April, 281-285.

13 Gojny, F. H., Nastalczyk, J., Roslaniec, Z., and Schulte, K. Surface modified multi-walled carbon nanotubes in
CNT/epoxy-composites. Chem. Phys. Lett., 2003, 370, 820-824.

14 Prato, M., Kostarelos, K., and Bianco, A. Functionalized carbon nanotubes in drug design and discovery. Acc. Chem. Res., 2008, 41, 60-68.

15 Vigolo, B., Pénicaud, A., Coulon, C., Sauder, C., Pailler, R., Journet, C., Bernier, P., and Poulin, P. Macroscopic fibers and ribbons of oriented carbon nanotubes. Science, 2000, 290, 1331-1334.

16 Safadi, B., Andrews, R., and Grulke, E. A. Multiwalled carbon nanotube polymer composites: synthesis and characterization of thin films. J. Appl. Polym. Sci., 2002, 84, 2660-2669.

17 Ericson, L. M., Fan, H., Peng, H., Davis, V. A., Zhou, W., Sulpizio, J., Wang, Y., Booker, R., Vavro, J., Guthy, C., Parra-Vasquez, A. N. G., Kim, M. J., Ramesh, S., Saini, R. K., Kittrell, C., Lavin, G., Schmidt, H., Adams, W. W., Billups, W. E., Pasquali, M., Hwang, W. F., Hauge, R. H., Fischer, J. E., and Smalley, R. E. Macroscopic, neat, single-walled carbon nanotube fibers. Science, 2004, 305, 1447-1450.

18 Kozlov, M. E., Capps, R. C., Sampson, W. M., Ebron, V. H., Ferraris, J. P., and Baughman, R. H. Spinning solid and hollow polymer-free carbon nanotube fibers. Adv. Mater., 2005, 17, 614-617.

19 Kordás, K., Mustonen, T., Tóth, G., Jantunen, H., Lajunen, M., Soldano, C., Talapatra, S., Kar, S., Vajtai, R., and Ajayan, P. M. Inkjet printing of electrically conductive patterns of carbon nanotubes. Small, 2006, 2, 1021-1025.

20 Kashiwagi, T., Du, F., Winey, K. I., Groth, K. M., Shields, J. R., Bellayer, S. P., Kim, H., and Douglas, J. F. Flammability properties of polymer nanocomposites with single-walled carbon nanotubes: effects of nanotube dispersion and concentration. Polymer, 2005, 46, 471-481.

21 Rahatekar, S. S., Hamm, M., Shaffer, M. S. P., and Elliot, J. A. Mesoscale modeling of electrical percolation in fiber-filled systems. J. Chem. Phys., 2005, 123, 134702-1-5.

22 Davis, V. A., Ericson, L. M., Parra-Vasquez, A. N. G., Fan, H., Wang, Y., Prieto, V., Longoria, J. A., Ramesh, S., Saini, R. K., Kittrell, C., Billups, W. E., Adams, W. W., Hauge, R. H., Smalley, R. E., and Pasquali, M. Phase behavior and rheology of SWNTs in superacids. Macromolecules, 2004, 37, 154-160.

23 Martin, C. A., Sandler, J. K. W., Windle, A. H., Schwarz, M.-K., Bauhofer, W., Schulte, K., and Shaffer, M. S. P. Electric field-induced aligned multi-wall carbon nanotube networks in epoxy composites. Polymer, 2005, 46, 877-886.

24 Toledo, N. C., De Planque, M. R. R., Antoranz Contera, S., Grobert, N., and Ryan, J. F. Lipidmodulated assembly of magnetized iron-filled carbon nanotubes in millimeter-scale structures. Jpn. J. Appl. Phys., 2007, 46, 2799-2805.

25 Rahatekar, S. S., Koziol, K. K. K., Butler, S. A., Elliott, J. A., Shaffer, M. S. P., Mackley, M. R., and Windle, A. H. Optical microstructure and viscosity enhancement for an epoxy resin matrix containing multi-wall carbon nanotubes. J. Rheol., 2006, 50, 599-610. 
26 Hobbie, E. K., Wang, H., Kim, H., Han, C. C., and Grulke, E. A. Optical measurements of structure and orientation in sheared carbon-nanotube suspensions. Rev. Sci. Instrum., 2003, 74, 1244-1250.

27 Hobbie, E. K., Wang, H., Kim, H., Lin-Gibson, S., and Grulke, E. A. Orientation of carbon nanotubes in a sheared polymer melt. Phys. Fluids, 2003, 15, 1196-1202.

28 Lin-Gibson, S., Pathak, J. A., Grulke, E. A., Wang, H., and Hobbie, E. K. Elastic flow instability in nanotube suspensions. Phys. Rev. Lett., 2004, 92, 048302-1-4.

29 Hobbie, E. K. and Fry, D. J. Nonequilibrium phase diagram of sticky nanotube suspensions. Phys. Rev. Lett., 2006, 97, 036101-1-4.

30 Song, W., Kinloch, I. A., and Windle, A. H. Nematic liquid crystallinity of multiwall carbon nanotubes. Science, 2003, 302, 1363.

31 Du, F., Scogna, R. C., Zhou, W., Brand, S., Fischer, J. E., and Winey, K. I. Nanotube networks in polymer nanocomposites: rheology and electrical conductivity. Macromolecules, 2004, 37, 9048-9055.

32 Mitchell, C. A., Bahr, J. L., Arepalli, S., Tour, J. M., and Krishnamoorti, R. Dispersion of functionalized carbon nanotubes in polystyrene. Macromolecules, 2002, 35, 8825-8830.

33 Hough, L. A., Islam, M. F., Janmey, P. A., and Yodh, A. G. Viscoelasticity of single wall carbon nanotube suspensions. Phys. Rev. Lett., 2004, 93, 168102-1-4.

34 Song, Y. S. and Youn, J. R. Influence of dispersion states of carbon nanotubes on physical properties of epoxy nanocomposites. Carbon, 2005, 43, 1378-1385.

35 Kim, B., Park, H., and Sigmund, W. M. Rheological behavior of multiwall carbon nanotubes with polyelectrolyte dispersants. Colloids Surf. A, 2005, 256, 123-127.

36 Schartel, B., Pötschke, P., Knoll, U., and Abdel-Goad, M. Fire behavior of polyamide 6/multiwall carbon nanotube nanocomposites. Eur. Polym. J., 2005, 41, 1061-1070.

37 Pötschke, P., Fornes, T. D., and Paul, D. R. Rheological behavior of multiwalled carbon nanotube/polycarbonate composites. Polymer, 2002, 43, 3247-3255.

38 Huang, Y. Y., Ahir, S. V., and Terentjev, E. M. Dispersion rheology of carbon nanotubes in a polymer matrix. Phys. Rev. B, 2006, 73, 125422-1-9.

39 McNally, T., Pötschke, P., Halley, P., Murphy, M., Martin, D., Bell, S. E. J., Brennan, G. P., Bein, D., Lemoine, P., and Quinn, J. P. Polyethylene multiwalled carbon nanotube composites. Polymer, 2005, 46, 82228232.

40 Hobbie, E. K. and Fry, D. J. Rheology of concentrated carbon nanotube suspensions. J. Chem. Phys., 2007, 126, 124907-1-7.

41 Kharchenko, S. B., Douglas, J. F., Obrzut, J., Grulke, E. A., and Migler, K. B. Flow-induced properties of nanotube-filled polymer materials. Nat. Mater., 2004, 3, 564-568.

42 Kinloch, I. A., Roberts, S. A., and Windle, A. H. A rheological study of concentrated aqueous nanotube dispersions. Polymer, 2002, 43, 7483-7491.

43 Lazano, K., Bonilla-Rios, J., and Barrera, E. V. A study on nanofibre-reinforced thermoplastic composites (II): investigation of the mixing rheology and conduction properties. J. Appl. Polym. Sci., 2001, 80, 1162-1172.
$44 \mathrm{Xu}$, J., Chatterjee, S., Koelling, K. W., Wang, Y., and Bechtel, S. E. Shear and extensional rheology of carbon nanofiber suspensions. Rheol. Acta, 2005, 44, 537-562.

45 Handge, U. A. and Pötschke, P. Deformation and orientation during shear and elongation of a polycarbonate/carbon nanotubes composite in the melt. Rheol. Acta, 2007, 46, 889-898.

46 Huang, Y. Y. and Terentjev, E. M. Dispersion and rheology of carbon nanotubes in polymers. Int. J. Mater. Form., 2008, 2, 63-74.

47 Fan, Z. and Advani, S. G. Rheology of multiwall carbon nanotube suspensions. J. Rheol., 2007, 51, 585-604.

48 Hong, J. S. and Kim, C. Extension-induced dispersion of multi-walled carbon nanotube in non-Newtonian fluid. J. Rheol., 2007, 51, 833-850.

49 Ma, A. W. K., Chinesta, F., and Mackley, M. R. The microstructure and rheology of carbon nanotube suspensions. In Science and applications of carbon nanotubes (Ed. M. Chipara), 2009, in press (Blackwell, London).

50 Kelemen, M. W. and Sharpe, J. E. Controlled cell disruption: a comparison of the forces required to disrupt different micro-organisms. J. Cell Sci., 1979, 35, 431-441.

51 Zhang, W. D., Shen, L., Phang, I. Y., and Liu, T. Carbon nanotubes reinforced Nylon-6 composite prepared by simple melt-compounding. Macromolecules, 2004, 37, 256-259.

52 Badaire, S., Poulin, P., Maugey, M., and Zakri, C. In situ measurements of nanotube dimensions in suspensions by depolarized dynamic light scattering. Langmuir, 2004, 20, 10367-10370.

53 Hennrich, F., Krupke, R., Arnold, K., Stutz, J. A. R., Lebedkin, S., Koch, T., Schimmel, T., and Kappes, M. M. The mechanism of cavitation-induced scission of single-walled carbon nanotubes. J. Phys. Chem. B, 2007, 111, 1932-1937.

54 Banerjee, S., Hemraj-Benny, T., and Wong, S. S. Covalent surface chemistry of single-walled carbon nanotubes. Adv. Mater., 2005, 17, 17-29.

55 Balasubramanian, K. and Burghard, M. Chemically functionalized carbon nanotubes. Small, 2005, 1, 180-192.

56 Murakami, H., Nomura, T., and Nakashima, N. Noncovalent porphyrin-functionalized single-walled carbon nanotubes in solution and the formation of porphyrinnanotube nanocomposites. Chem. Phys. Lett., 2003, 378, 481-485.

57 Ham, H. T., Choi, Y. S., and Chung, I. J. An explanation of dispersion states of single-walled carbon nanotubes in solvents and aqueous surfactant solutions using solubility parameters. J. Colloid Interface Sci., 2005, 286, 216-223.

58 Ma, A., Lu, J., Yang, S., and Ng, K. M. Quantitative non-covalent functionalization of carbon nanotubes. J. Cluster Sci., 2006, 17, 599-608.

59 Ma, A. W. K. The microstructure and rheology of carbon nanotube suspensions. PhD Thesis, Department of Chemical Engineering and Biotechnology, University of Cambridge, 2008. 
60 Ma, A. W. K., Chinesta, F., Ammar, A., and Mackley, M. R. Rheological modelling of carbon nanotube aggregate suspensions. J. Rheol., 2008, 52, 1311-1330.

61 Donald, A. M., Windle, A. H., and Hanna, S. Liquid crystalline polymers, 2006 (Cambridge University Press, Cambridge).

62 Zasadzinski, J. A. N. and Meyer, R. B. Molecular imaging of tobacco mosaic virus lyotropic nematic phases. Phys. Rev. Lett., 1986, 56, 636-638.

63 Ma, A. W. K., Mackley, M. R., and Rahatekar, S. S. Experimental observation on the flow-induced assembly of carbon nanotube suspensions to form helical bands. Rheol. Acta, 2007, 46, 979-987.

64 Mason, S. G. The flocculation of cellulose fibre suspensions. Pulp Paper Mag. Can., 1948, 49, 99-104.

65 Mason, S. G. The flocculation of pulp suspensions and the formation of paper. Tappi J., 1950, 33, 440-444.

66 Switzer, L. H. and Klingenberg, D. J. Flocculation in simulations of sheared fiber suspensions. Int. J. Multiphase Flow, 2004, 30, 67-87.

67 Schmidt, R. H., Kinloch, I. A., Burgess, A. N., and Windle, A. H. The effect of aggregation on the electrical conductivity of spin-coated polymer/carbon nanotube composite films. Langmuir, 2007, 23, 5707-5712.

68 Yamamoto, K., Akita, S., and Nakayama, Y. Orientation of carbon nanotubes using electrophoresis. Jpn. J. Appl. Phys., 1996, 35, L917-L918.

69 Yamamoto, K., Akita, S., and Nakayama, Y. Orientation and purification of carbon nanotubes using ac electrophoresis. J. Phys. D: Appl. Phys., 1998, 31, L34-L36.

70 Bubke, K., Gnewuch, H., Hempstead, M., Hammer, J., and Green, M. L. H. Optical anisotropy of dispersed carbon nanotubes induced by an electric field. Appl. Phys. Lett., 1997, 71, 1906-1908.

71 Chen, X. Q., Saito, T., Yamada, H., and Matsushige, K. Aligning single-wall carbon nanotubes with an alternating-current electric field. Appl. Phys. Lett., 2001, 78, 3714-3716.

72 Nagahara, L. A., Amlani, I., Lewenstein, J., and Tsui, R. K. Directed placement of suspended carbon nanotubes for nanometer-scale assembly. Appl. Phys. Lett., 2002, 80, 3826-3829.

73 Krupke, R., Hennrich, F., Löhneysen, H. V., and Kappes, M. M. Separation of metallic from semiconducting single-walled carbon nanotubes. Science, 2003, 301, 344-347.

74 Fujiwara, M., Oki, E., Hamada, M., Tanimoto, Y., Mukouda, I., and Shimomura, Y. Magnetic orientation and magnetic properties of a single carbon nanotube. J. Phys. Chem. A, 2001, 105, 4383-4386.

75 Kimura, T., Ago, H., Tobita, M., Ohshima, S., Kyotani, M., and Yumura, M. Polymer composites of carbon nanotubes aligned by a magnetic field. Adv. Mater., 2002, 14, $1380-1383$.

76 Islam, M. F., Milkie, D. E., Torrens, O. N., Todh, A. G., and Kikkawa, J. M. Magnetic heterogeneity and alignment of single wall carbon nanotubes. Phys. Rev. B, 2005, 71, 201401-1-4.

77 Céspedes, O., Ferreira, M. S., Sanvito, S., Kociak, M., and Coey, J. M. D. Contact induced magnetism in carbon nanotubes. J. Phys.: Condens. Matter, 2004, 16, L155-L161.
78 Samouhos, S. and McKinley, G. Carbon nanotubemagnetite composites, with applications to developing unique magnetorheological fluids. J. Fluids Engng., 2007, 129, 429-437.

79 Yang, Z.-P., Ci, L., Bur, J. A., Lin, S.-Y., and Ajayan, P. M. Experimental observation of an extremely dark material made by a low-density nanotube array. Nano Lett., 2008, 8, 446-451.

80 Fry, D., Langhorst, B., Kim, H., Grulke, E., Wang, H., and Hobbie, E. K. Anisotropy of sheared carbonnanotube suspensions. Phys. Rev. Lett., 2005, 95, 038304-1-4.

81 Rao, A. M., Jorio, A., Pimenta, M. A., Dantas, M. S. S., and Saito, R. Polarized Raman study of aligned multiwalled carbon nanotubes. Phys. Rev. Lett., 2000, 84, 1820-1823.

82 Park, C., Wilkinson, J., Banda, S., Ounaies, Z., Wise, K. E., Sauti, G., Lillehei, P. T., and Harrison, J. S. Aligned singlewall carbon nanotube polymer composites using an electric field. J. Polym. Sci., Part B: Polym. Phys., 2006, 44, 1751-1762.

83 Motta, M., Moisala, A., Kinloch, I. A., and Windle, A. H. High performance fibres from 'dog bone' carbon nanotubes. Adv. Mater., 2007, 19, 3712-3726.

84 Shaffer, M. S. P., Fan, X., and Windle, A. H. Dispersion and packing of carbon nanotubes. Carbon, 1998, 36, 1603-1612.

85 Ahir, S. V., Squires, A. M., Tajbakhsh, A. R., and Terentjev, E. M. Infrared actuation in aligned polymernanotube composites. Phys. Rev. B, 2006, 73, 085420$1-12$.

86 Tsyboulski, D. A., Bachilo, S. M., and Weisman, R. B. Versatile visualization of individual single-walled carbon nanotubes with near-infrared fluorescence microscopy. Nano Lett., 2005, 5, 975-979.

87 Otobe, K., Nakao, H., Hayashi, H., Nihey, F., Yudasaka, M., and Iijima, S. Fluorescence visualization of carbon nanotubes by modification with silicon-based polymer. Nano Lett., 2002, 2, 1157-1160.

88 Prakash, R., Washburn, S., Superfine, R., Cheney, R. E., and Falvo, M. R. Visualization of individual carbon nanotubes with fluorescence microscopy using conventional fluorophores. Appl. Phys. Lett., 2003, 83, 1219-1221.

89 Chaudhary, S., Kim, J. H., Singh, K., and Ozkan, M. Fluorescence microscopy visualization of single-walled carbon nanotubes using semiconductor nanocrystals. Nano Lett., 2004, 4, 2415-2419.

90 Duggal, R. and Pasquali, M. Dynamics of individual single-walled carbon nanotubes in water by real-time visualization. Phys. Rev. Lett., 2006, 96, 246104-1-4.

91 Fan, Z. and Advani, S. G. Characterization of orientation state of carbon nanotubes in shear flow. Polymer, 2005, 46, 5232-5240.

92 Ma, A. W. K., Mackley, M. R., and Chinesta, F. The microstructure and rheology of carbon nanotube suspensions. Int. J. Mater. Form., 2008, 2, 75-81.

93 Cross, M. M. Rheology of non-Newtonian fluids: a new flow equation for pseudoplastic systems. J. Colloid Sci., 1965, 20, 417-437. 
94 Ma, A. W. K., Chinesta, F., and Mackley, M. R. The rheology and modelling of chemically treated carbon nanotube suspensions. J. Rheol., 2009, 53, 547-573.

95 Litchfield, D. W. and Baird, D. G. The rheology of high aspect ratio nano-particle filled liquids. In Rheology reviews (Eds D. M. Binding and K. Walters), 2006, pp. 1-60 (The British Society of Rheology).

96 Flory, P. J. Molecular size distribution in three dimensional polymers. I. Gelation. J. Am. Chem. Soc., 1941, 63, 3083-3090.

97 Flory, P. J. Constitution of three-dimensional polymers and the theory of gelation. J. Phys. Chem., 1942, 46, $132-140$.

98 Stockmayer, W. H. Theory of molecular size distribution and gel formation in branched-chain polymers. J. Chem. Phys., 1943, 11, 45-55.

99 Stauffer, D. Gelation in concentrated critically branched polymer solutions. J. Chem. Soc. Faraday Trans. II, 1976, 72, 1354-1364.

100 de Gennes, P. G. On a relation between percolation theory and the elasticity of gels. J. Phys. (Paris), 1976, 37, L-1-2.

101 Winter, H. H. Soft polymeric materials near the transition from liquid to solid state. Korea-Aust. Rheol. J., 1999, 11, 275-278.

102 Chambon, F. and Winter, H. H. Stopping of crosslinking reaction in a PDMS polymer at the gel point. Polym. Bull., 1985, 13, 499-503.

103 Chambon, F. and Winter, H. H. Linear viscoelasticity at the gel point of a crosslinking PDMS with imbalanced stoichiometry. J. Rheol., 1987, 31, 683-698.

104 Winter, H. H. and Chambon, F. Analysis of linear viscoelasticity of a crosslinking polymer at the gel point. J. Rheol., 1986, 30, 367-382.

105 Mours, M. and Winter, H. H. Relaxation patterns of nearly critical gels. Macromolecules, 1996, 29, 72217229 .

106 Mewis, J. and Macosko, C. W. Suspension rheology. In Rheology: Principles, measurements, and applications (Ed. C. W. Macosko), 1994, pp. 435-470 (Wiley, New York, New York).

107 Mewis, J. and Meire, C. Yielding in weakly flocculated systems. Fluids (Eds B. Mena, A. García-Rejón and C. Rangel-Nafaille), in Advances in rheology series, vol. 2, 1984, pp. 591-598 (Elsevier, Amsterdam).

108 Amari, T. and Watanabe, K. Stress relaxation of carbon black-linseed oil suspensions. J. Soc. Rheol. Jpn., 1980, 8, 80-83.

109 Guskey, S. M. and Winter, H. H. Transient shear behavior of a thermotropic liquid crystalline polymer in the nematic state. J. Rheol., 1991, 35, 1191-1207.

110 Mackley, M. R., Marshall, R. T. J., Smeulders, J. B. A. F., and Zhao, F. D. The rheological characterization of polymeric and colloidal fluids. Chem. Engng Sci., 1994, 49, 2551-2565.

111 Wagner, M. H. Analysis of time-dependent non-linear stress growth data for shear and elongational flow of a low-density branched polyethylene melt. Rheol. Acta, 1976, 15, 136-142.

112 Song, J.-W., Kim, J., Yoon, Y.-H., Choi, B.-S., Kim, J.-H., and Han, C.-S. Inkjet printing of single-walled carbon nanotubes and electrical characterization of the line pattern. Nanotechnology, 2008, 19, 095702$1-6$.

113 Jäder, J., Willenbacher, N., Engström, G., and Järnström, L. The influence of extensional properties on the dewatering of coating colours. J. Pulp Paper Sci., 2005, 31, 181-187.

114 Xu, J., Chatterjee, S., Koelling, K. W., Wang, Y., and Bechtel, S. E. Shear and extensional rheology of carbon nanofiber suspensions. Rheol. Acta, 2005, 44, 537-562.

115 Ma, A. W. K., Chinesta, F., Tuladhar, T., and Mackley, M. R. Filament stretching of carbon nanotube suspensions. Rheol. Acta, 2008, 47, 447-457.

116 Weinberger, G. B. and Goddard, J. D. Extensional flow behaviour of polymer solutions and particle suspensions in spinning motion. Int. J. Multiphase Flow, 1974, 1, 465-486.

117 Abbasi, S., Derdouri, A., and Carreau, P. J. Rheology, morphology and temperature dependence of nanotube networks in polycarbonate/multiwalled carbon nanotube composites. In Proceedings of the XVth International Congress on Rheology, Monterey, California, 3-8 August 2008, pp. 499-501 (The Society of Rheology).

118 Pötschke, P. Melt processing of multiwalled carbon nanotube-polymer composites. In Proceedings of the 2nd Annual Symposium on The science and application of carbon nanotube and related structures, Churchill College, Cambridge, December 2008 (Cambridge CNT Society).

119 Ausman, K. D., Piner, R., Lourie, O., and Ruoff, R. S. Organic solvent dispersions of single-walled carbon nanotubes: toward solutions of pristine nanotubes. J. Phys. Chem. B, 2000, 104, 8911-8915.

120 Martin, C. A., Sandler, J. K. W., Shaffer, M. S. P., Schwarz, M.-K., Bauhofer, W., Schulte, K., and Windle, A. H. Formation of percolating networks in multi-wall carbon-nanotube-epoxy composites. Compos. Sci. Technol., 2004, 64, 2309-2316.

121 Bartram, E., Goldsmith, H. L., and Mason, S. G. Particle motions in non-Newtonian media III. Further observations in elasticoviscous fluids. Rheol. Acta, 1975, 14, 776-782.

122 Johnson, S. J., Salem, A. J., and Fuller, G. G. Dynamics of colloidal particles in sheared, non-Newtonian fluids. J. Non-Newtonian Fluid Mech., 1990, 34, 89-121.

123 Iso, Y., Cohen, C., and Koch, D. L. Orientation in simple shear flow of semi-dilute fiber suspensions 2 . Highly elastic fluids. J. Non-Newtonian Fluid Mech., 1996, 62, 135-153.

124 Iso, Y., Koch, D. L., and Cohen, C. Orientation in simple shear flow of semi-dilute fiber suspensions 1 . Weakly elastic fluids. J. Non-Newtonian Fluid Mech., 1996, 62, 115-134.

125 Rahatekar, S. S., Koziol, K. K., Kline, S. R., Hobbie, E. K., Gilman, J. W., and Windle, A. H. Lengthdependent mechanics of carbon-nanotube networks. Adv. Mater., 2009, 21, 874-878.

126 Hobbie, E. K. Rheology of carbon nanotube suspensions and networks. In Proceedings of the XVth International Congress on Rheology, Monterey, California, 3-8 August 2008 (The Society of Rheology). 
127 Abdel-Goad, M. and Pötschke, P. Rheological characterization of melt processed polycarbonate-multiwalled carbon nanotube composites. J. Non-Newtonian Fluid Mech., 2005, 128, 2-6.

128 Doi, M. and Edwards, S. F. The theory of polymer dynamics, 1986 (Oxford Press, Oxford).

129 Folgar, F. and Tucker, C. L. III Orientation behaviour of fibers in concentrated suspensions. J. Reinf. Plast. Compos., 1984, 3, 98-119.

130 Laso, M., Muneta, L. M., Müller, V., Alcazar, A., Chinesta, F., and Ammar, A. Hierarchical approach to flow calculations for polymeric liquid crystals. In Multiscale modelling of polymer properties (Eds E. A. Perpète and M. Laso), 2006, pp. 359-402 (Elsevier, Amsterdam).

131 Leonov, A. I. On the rheology of filled polymers. J. Rheol., 1990, 34, 1039-1068.

132 Dinsmore, A. D., Prasad, V., Wong, I. Y., and Weitz, D. A. Microscopic structure and elasticity of weakly aggregated colloidal gels. Phys. Rev. Lett., 2006, 96, 185502.

133 Vaccaro, A. and Marrucci, G. A model for the nonlinear rheology of associating polymers. J. NonNewtonian Fluid Mech., 2000, 92, 261-273.
134 Hernandez Cifre, J. G., Barenbrug, Th. M. A. O. M., Schieber, J. D., and van den Brule, B. H. A. A. Brownian dynamics simulation of reversible polymer networks under shear using a non-interacting dumbbell model. J. Non-Newtonian Fluid Mech., 2003, 113, 73-96.

135 Rincón, E., Chávez, A. E., Herrera, R., and Manero, 0. Rheological modelling of complex fluids: a transient network model with microstates. J. Non-Newtonian Fluid Mech., 2005, 131, 64-77.

136 Song, Y. S. and Youn, J. R. Modeling of rheological behaviour of nanocomposites by Brownian dynamics simulation. Korea-Aust. Rheol. J., 2004, 16, 201-212.

137 Qiu, J., Zhang, C., Wang, B., and Liang, R. Investigation of the flow behaviours of carbon nanotubes dispersed epoxy resin with modified bi-mode FENE dumbbell simulation. Compos. Mater. Sci., 2009, 44, 1379-1385.

138 Cueto, E., Ma, A. W. K., Chinesta, F., and Mackley, M. R. Numerical simulation of spin coating processes involving functionalised carbon nanotube suspensions. Int. J. Mater. Form., 2008, 2, 89-99.

139 Petrie, C. J. S. The rheology of fibre suspensions. J. Non-Newtonian Fluid Mech., 1999, 87, 369-402. 